\title{
Identification and Distribution of Botryosphaeria spp. Associated with Grapevine Cankers in California
}

\author{
J. R. Úrbez-Torres, Department of Plant Pathology, University of California, Davis 95616; G. M. Leavitt, Univer- \\ sity of California Cooperative Extension, Madera 93637; and T. M. Voegel and W. D. Gubler, Department of Plant \\ Pathology, University of California, Davis
}

\begin{abstract}
Úrbez-Torres, J. R., Leavitt, G. M., Voegel, T. M., and Gubler, W. D. 2006. Identification and distribution of Botryosphaeria spp. associated with grapevine cankers in California. Plant Dis. 90:1490-1503.

Botryosphaeria spp. recently have been identified as important grapevine pathogens worldwide. To date, Botryosphaeria rhodina has been the only species associated with cankers on Vitis vinifera in California. A field survey of 166 vineyards in 21 counties was conducted in order to determine the occurrence of other Botryosphaeria spp. in California. In all, 1,735 samples of cankered trunks, cordons, and spurs were collected. Botryosphaeria spp. were the most common fungi isolated from grapevine cankers in California. Morphological identification along with phylogenetic analysis of the internal transcribed spacer region (ITS1-5.8S-ITS2) of the nuclear ribosomal DNA (rDNA) and a partial sequence of the $\beta$-tubulin gene showed that at least seven Botryosphaeria spp. occur on grapevines in California: B. australis, B. dothidea, B. lutea, $B$. obtusa, B. parva, B. rhodina, and B. stevensii. Botryosphaeria spp. were found in grapevine cankers in all grape-growing regions surveyed in California, whereas incidence and distribution varied with location. Grapevine cankers in California have been associated mainly with Eutypa dieback. However, the frequent recovery of Botryosphaeria spp. from cankers in this study indicates that the role of these fungi in grapevine health needs to be more carefully considered.
\end{abstract}

Additional keywords: esca, Eutypa lata, Phomopsis viticola, trunk diseases

The California grapevine industry (wine, raisin, and table grapes) presently comprises over 345,200 ha and is the third most important agricultural commodity in the state, producing an annual crop valued at over $\$ 2.3$ billion (40). Fungal trunk diseases have become a growing threat to grapevines in California and throughout the world. Trunk diseases of grapevine are caused by fungal pathogens that invade through pruning wounds located on the woody parts of the plant. Symptoms include a slow decline and dieback of the vine as a result of interruption of xylem conductivity or toxin production (19). Trunk diseases principally include black measles (esca), Eutypa dieback, young vine decline, and other diseases caused by Botryosphaeria spp. (19). Trunk diseases reduce yields and increase production costs. Production costs are increased as a result of cultural and chemical preventive

Corresponding author: W. D. Gubler

E-mail: wdgubler@ucdavis.edu

This work was partially funded by grants from the American Vineyard Foundation and the Viticultural Consortium.

Accepted for publication 20 June 2006.

DOI: 10.1094/PD-90-1490

(C) 2006 The American Phytopathological Society measures as well as removing diseased wood from the vine after infection (9). In California, the overall loss in net income for wine grape caused by Eutypa dieback and Botryosphaeria canker disease was estimated to be over $\$ 260$ million per annum (32).

Botryosphaeria spp. occur on a large number of hosts and have a wide geographical distribution $(26,42)$. Since this genus was introduced in 1863 (Ces. \& De Not.), different species of Botryosphaeria have been well established as cankercausing agents in several woody plants $(26,27)$. However, their association with dieback in grapevine has been overlooked for many years because Botryosphaeria spp. have been largely considered saprophytes or secondary colonizers in grapevines (25). To date, 13 Botryosphaeria spp. have been reported to cause disease symptoms in grapevines in different production regions worldwide, but only in the last decade has the significance of these fungi as grapevine pathogens been recognized $(25,37,41)$. Botryosphaeria spp. known to occur in grapevine include $B$. australis Slippers, Crous, \& M. J. Wingf., B. dothidea (Moug.:Fr.) Ces. \& De Not., $B$. lutea A. J. L. Phillips, B. obtusa (Schwein.) Shoemaker, B. parva Pennycook \& Samuels, B. rhodina von Arx, $B$. ribis Gross. \& Duggar, B. stevensii Shoemaker, B. viticola A. J. L. Phillips \& Lu- que, and the anamorphs Diplodia sarmentorum (Fr.:Fr.) Fr., D. porosum Niekerk \& Crous, Fusicoccum viticlavatum Niekerk \& Crous, and F. vitifusiforme Niekerk \& Crous $(16,25,41)$.

Botryosphaeria spp. frequently have been isolated from grapevines showing decline or dieback symptoms in Egypt (5), California $(9,14)$, Arizona, Mexico (13), Hungary (15), France (12), Italy (29), Portugal (23-25), Spain $(16,39)$, South Africa (41), Chile (1), and Australia (2,37). Pathogenicity and epidemiology of Botryosphaeria spp. in vines have been the subject of confusion for many years $(25,41)$. Virulence and symptoms caused by Botryosphaeria spp. on grapevines have been reported to be different depending on cultivars and countries. For example, $B$. dothidea, B. obtusa, and B. stevensii were found to be the cause of "black dead arm" in France. The disease was characterized by wood streaking and red patches at the margin of the leaves, with large areas of chlorosis and deterioration between the veins (12). In contrast, no symptoms of black dead arm were found associated with the same species on grapevines in Portugal $(23,25)$.

The best example of controversy concerning pathogenicity of Botryosphaeria spp. occurs with $B$. obtusa. Although this species has been reported as virulent on grapevine in Chile (1), New South Wales region in Australia (2), and South Africa (41), it has been considered weakly pathogenic in Portugal (25). In another study, $B$. obtusa did not cause any symptom on inoculated grapevine cuttings in Western Australia (37). Several vascular and foliar symptoms attributed to Botryosphaeria spp. in grapevine, such as perennial cankers, trunk dieback, wood necrosis, vascular streaking and mild chlorosis, or wilting of the leaves, have been described $(12,25,41)$, but they often are difficult to distinguish from symptoms caused by other fungal pathogens, such as Eutypa lata (Pers.:Fr.) Tul. \& C. Tul. (2), E. leptoplaca (Mont.) Rappaz (38), and Phomopsis viticola (Sacc.) Sacc. $(2,24)$. Thus, the relationships between Botryosphaeria spp. and grapevine disease symptoms have been difficult to determine.

To date, grapevine trunk diseases in California have been associated mainly with E. lata, Phaeomoniella chlamydospora, and different species of Phaeo- 
acremonium causing Eutypa dieback, Petri disease, and esca (black measles), respectively $(6,19,31,32)$. New findings also have shown two species of Cylindrocarpon to be the cause of black foot disease of Californian grapevines (21). However, the role of Botryosphaeria spp. on grapevines in the state has remained largely unknown. Of the Botryosphaeria spp. that are recognized to occur on grapevine worldwide, $B$. rhodina was the only species reported from Californian grapevines $(14,35,43)$. It is associated with wedge-shaped cankers and grapevine dieback in California and the disease it causes has been known locally as "Bot canker." B. rhodina is now considered an endemic pathogen in arid climate regions of southern California (10).

The objectives of this study were to (i) identify the species of Botryosphaeria associated with canker symptoms in Californian grapevines based on morphological and molecular characteristics and (ii) determine their geographic distribution in California vineyards.

\section{MATERIALS AND METHODS}

Field survey, fungal isolation, and data analysis. Between 10 and 15 perennial cankers from spurs, cordons, or trunks were collected from different grapevines in each surveyed vineyard (Fig. 1a-c). In all, 1,735 samples were collected from 166 vineyards in 21 counties throughout California between 2003 and 2005. Samples were obtained from 111 and 55 vineyards from the predominant wine and tableraisin grape cultivars of California, respectively. Grapevine samples were cleaned of loose bark and surface disinfested in $10 \%$ sodium hypochlorite for $10 \mathrm{~min}$. After air drying, the surface tissue was cut away to expose both sides of the canker. Small pieces of tissue from both sides of the canker were placed on 85-mm-diameter petri dishes containing $4 \%$ potato dextrose agar (PDA) (Difco Laboratories, Detroit) amended with tetracycline hydrochloride $(0.01 \%)$ (Sigma-Aldrich, St. Louis) (PDAtet). Cultures were incubated at room temperature until fungal colonies were observed. Pure cultures of Botryosphaeria spp. were obtained by excising a hyphal tip from colony margins emerging from the tissue pieces onto fresh plates of PDA-tet.

Statistical analysis was performed in order to determine the relationship between the incidence of Botryosphaeria spp. and grapevine regions, grapevine age, and grapevine cultivar. Data were analyzed using analysis of variance (ANOVA) on the proportion of samples yielding Botryosphaeria spp. from each vineyard (ANOVA using PROC GLM, SAS System, version 8.1; SAS Institute, Cary, NC). To satisfy the assumptions of the ANOVA, the arcsine transformation of the proportion was used $(Y=2 \times \operatorname{arcsine} \sqrt{p})$. Homogeneity of variance was assessed using Levene's test. Tukey's test was used for treatments means comparisons. Vineyards from 21 counties were split into eight grapevine regions (Table 1 ).

Morphological characterization. Botryosphaeria spp. isolated from cankers were identified tentatively based on colony morphology and conidial characteristics reported in previous studies $(20,25,33,37)$. Botryosphaeria isolates first were divided based on colony morphology. The color and growth from these isolates were recorded during 15 days of growth on PDAtet at $25^{\circ} \mathrm{C}$ in a 12 -h light-and-dark cycle, illuminated by fluorescent and nearultraviolet light $(366 \mathrm{~nm})$. Characteristics of conidial morphology in 30 Botryosphaeria isolates also were observed after placing cultures on 2\% water agar (Difco Laboratories) containing autoclaved grapevine wood chips. Isolates were incubated at $25^{\circ} \mathrm{C}$ under intermittent light $(12 \mathrm{~h})$ for 5 weeks to induce sporulation (41). The length and width of 60 conidia per isolate were measured with the aid of a compound microscope (Nikon Inc. Instruments Group,
Melville, NY) using the imaging device SPOT RT software (v3.5.1, SPOT; Diagnostic Instruments Inc., MI). Mean and standard deviation of the conidial measurements were obtained using summary statistics in SAS and compared with those previously reported for Botryosphaeria spp. Conidial color, shape, and presence or absence of septation also were recorded.

Botryosphaeria isolates selected for conidial characterization also were used to determine the effect of temperature on colony growth. A 5-mm-diameter plug from the growing margin of a 3-day-old colony was placed in the center of an 85mm-diameter PDA-tet petri dish, and three replicates of each isolate were incubated separately at $5,10,15,20,25,30,35$, and $40^{\circ} \mathrm{C}$ in the dark. Colony diameter was measured after 2, 3, and 4 days of incubation, and data were converted to radial growth in millimeters (30). The experiment was conducted twice. Radial growth values after $48 \mathrm{~h}$ versus temperature were adjusted to regression curves using the Rat-

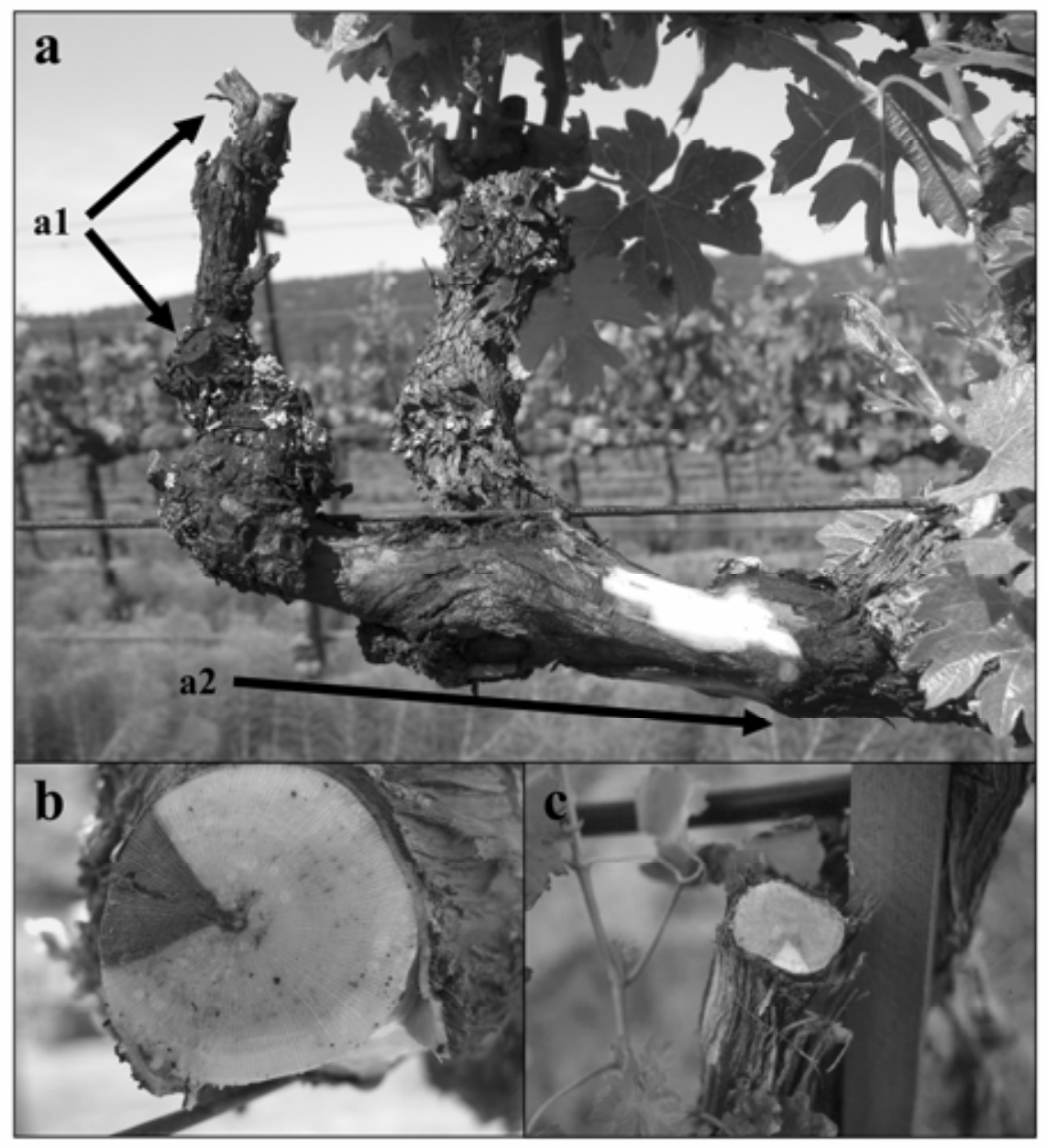

Fig. 1. a, Dead spur position and perennial developing canker in an 18-year-old Merlot grapevine. a1, Infection starts when conidia or ascospores colonize fresh pruning wounds. a2, Lengthwise canker growth develops slowly from the point of infection basipetally. b, Cross section of a 12-year-old Zinfandel grapevine cordon. Wedge-shaped canker is the typical symptom in the earliest stages of the disease. c, Mature canker in the trunk of a 17-year-old Cabernet Sauvignon grapevine. Perennial cankers also grow in a lateral direction in the spurs, cordons, and trunks of the vine parts for several years until only a small wedge of tissue remains alive. Death of the vines parts occurs when the remaining tissue is killed by the growth of the fungus. 
kowsky model (28); a four-parameter model fit the values in the regression model $\sqrt{r}=b\left(T-T_{\min }\right)(1-\exp [c(T-$ $\left.\left.T_{\max }\right)\right]$ ), where $\sqrt{r}$ is the square root of the radial growth, $b$ is a regression coefficient of the square root of growth rate constant versus degrees Kelvin for temperatures below the optimal temperature, $T_{\min }$ and $T_{\max }$ are the minimum and maximum temperatures, respectively, at which the rate of growth is zero, whereas $c$ is an additional parameter to enable the model to fit the data for temperatures above the optimal temperature (28). Nonlinear least squares were used to obtain the regression curves and optimum temperatures were calculated using numerical maximization (analyses performed using $\mathrm{R}$ version 2.2.0; Free Software Foundation, Inc., MA). Optimum temperature was defined as the temperature that produced maximum radial growth. ANOVA was used to determine the effects of radial growth difference by species at the optimum temperature after $48 \mathrm{~h}$.
DNA isolation, amplification, and phylogenetic analyses. Total genomic DNA from the Botryosphaeria isolates selected for morphology study was extracted from pure cultures as described by Cenis (3) (Table 2). Once DNA was obtained, oligonucleotide primers ITS4 and ITS5 were used to amplify and sequence the internal transcribed spacer (ITS) region, including the 5.8S (44). Partial sequence of the $\beta$-tubulin (BT) gene, BT2, was amplified using primers $\mathrm{Bt} 2 \mathrm{a}$ and $\mathrm{Bt} 2 \mathrm{~b}$ (8). Polymerase chain reaction (PCR) reactions were carried out in a thermal cycler (PTC 200; M. J. Research Company, Watertown, MA) as follows: an initial preheat for $2 \mathrm{~min}$ at $94^{\circ} \mathrm{C}$, followed by 35 cycles of denaturation at $94^{\circ} \mathrm{C}$ for $60 \mathrm{~s}$, annealing at $58^{\circ} \mathrm{C}$ for $60 \mathrm{~s}$, and extension at $72^{\circ} \mathrm{C}$ for $90 \mathrm{~s}$. The PCR amplification products were separated by electrophoresis in $1.2 \%$ agarose gels in $1.0 \times$ Tris-boric acid-EDTA (TBE) buffer and photographed after staining with ethidium bro- mide for $10 \mathrm{~min}$. PCR products were purified using the QIAquick PCR purification kit (Qiagen Inc., Valencia, CA). ITS and $\beta$ tubulin regions were sequenced in both directions by the University of California, Davis, Division of Biological Sciences (DBS) sequencing facility.

Sequences were edited using the software program Sequencher (version 4.1; Gene Codes, Ann Arbor, MI) and manually aligned using the computer software BioEdit sequencer alignment editor (version 7.0.0; Tom Hall, Isis Pharmaceuticals, Inc., Carlsbad, CA). Alignment gaps were treated as missing data. Whenever possible, sequences of Botryosphaeria spp. from grapevine and other hosts from previous studies available in GenBank were included in our analyses (Table 3). Separate phylogenetic analyses were run for the ITS dataset alone, $\beta$-tubulin dataset alone, and combined ITS and $\beta$-tubulin datasets using PAUP (version 4.0b10; Sinauer Associates, Inc., Sunderland, MA; 36). Guig-

Table 1. Incidence of Botryosphaeria spp. in the main grapevine-production areas of California

\begin{tabular}{|c|c|c|c|c|c|c|c|c|}
\hline \multirow[b]{2}{*}{ Grapevine region } & \multirow[b]{2}{*}{ Vineyards $^{\mathbf{b}}$} & \multirow[b]{2}{*}{ No. $(\%)$ Bot. $^{\text {c }}$} & \multirow[b]{2}{*}{ Total $^{d}$} & \multicolumn{5}{|c|}{ Number $(\%)$ of cankers yielding ${ }^{a}$} \\
\hline & & & & $\mathbf{A}^{\mathbf{e}}$ & $\mathbf{B}^{\mathbf{f}}$ & $\mathbf{A}+\mathbf{B}^{\mathbf{g}}$ & E. lata & P. viticola \\
\hline \multicolumn{9}{|l|}{ North Coast } \\
\hline Mendocino Co. & 8 & $8(100)$ & 91 & $37(41)$ & $11(12)$ & $48(53)$ & $11(12)$ & 0 \\
\hline Napa Co. & 11 & $10(91)$ & 113 & $30(26)$ & $13(11)$ & $43(38)$ & $54(48)$ & 0 \\
\hline Sonoma Co. & 17 & $17(100)$ & 192 & 85 (44) & $13(7)$ & $98(51)$ & $41(21)$ & 0 \\
\hline Total & 36 & $35(97)$ & 396 & $152(38)$ & $37(9)$ & $189(48)$ & $106(27)$ & 0 \\
\hline \multicolumn{9}{|l|}{ Central Coast } \\
\hline Santa Clara Co. & 2 & $2(100)$ & 23 & $10(43 \%)$ & $7(30)$ & $17(74)$ & $4(17)$ & 0 \\
\hline San Benito Co. & 6 & $6(100)$ & 65 & $49(75 \%)$ & $9(14)$ & $58(89)$ & $6(9)$ & $1(1)$ \\
\hline Monterey Co. & 6 & $6(100)$ & 65 & $44(68)$ & $4(6)$ & $48(74)$ & $13(20)$ & 0 \\
\hline San Luis Obispo Co. & 8 & $8(100)$ & 80 & 37 (46) & $7(9)$ & $44(55)$ & $15(19)$ & 0 \\
\hline Total & 22 & $22(100)$ & 233 & $140(60)$ & $27(12)$ & $167(72)$ & $38(16)$ & $1(0.5)$ \\
\hline \multicolumn{9}{|l|}{ South Coast } \\
\hline Santa Barbara Co. & 7 & $7(100)$ & 81 & $58(72)$ & $16(20)$ & $74(91)$ & $1(1)$ & $3(4)$ \\
\hline \multicolumn{9}{|l|}{ Mountain Counties } \\
\hline El Dorado Co. & 2 & $2(100)$ & 20 & $9(45)$ & $2(10)$ & $11(55)$ & $2(10)$ & 0 \\
\hline Amador Co. & 3 & $3(100)$ & 32 & $13(41)$ & $4(12)$ & $17(53)$ & $1(3)$ & $1(3)$ \\
\hline Total & 5 & $5(100)$ & 52 & $22(42)$ & $6(11)$ & $28(54)$ & $3(6)$ & $1(2)$ \\
\hline \multicolumn{9}{|l|}{ Sacramento Valley } \\
\hline Yolo Co. & 4 & $4(100)$ & 42 & $10(24)$ & $11(26)$ & $21(50)$ & $9(21)$ & 0 \\
\hline Solano Co. & 5 & $5(100)$ & 53 & $2(4)$ & $11(21)$ & $13(24)$ & $39(74)$ & 0 \\
\hline Sacramento Co. & 7 & 7 (100) & 78 & $35(45)$ & $28(36)$ & $63(81)$ & $11(14)$ & $3(4)$ \\
\hline Total & 16 & $16(100)$ & 173 & $47(27)$ & $50(29)$ & $97(56)$ & $59(34)$ & $3(2)$ \\
\hline \multicolumn{9}{|l|}{ Northern San Joaquin Valley } \\
\hline San Joaquin Co. & 7 & $5(71)$ & 65 & $4(6)$ & $6(9)$ & $10(15)$ & $34(52)$ & 0 \\
\hline Stanislaus Co. & 5 & $5(100)$ & 62 & $20(32)$ & $6(10)$ & $26(42)$ & $29(47)$ & 0 \\
\hline Merced & 10 & $10(100)$ & 116 & $63(54)$ & $21(18)$ & $84(72)$ & $4(3)$ & $1(1)$ \\
\hline Total & 22 & $20(91)$ & 243 & $87(36)$ & $33(13)$ & $120(49)$ & $67(27)$ & $1(0.5)$ \\
\hline \multicolumn{9}{|l|}{ Southern San Joaquin Valley } \\
\hline Madera Co. & 18 & $10(55)$ & 198 & $27(14)$ & $5(2)$ & $32(16)$ & $17(9)$ & $16(8)$ \\
\hline Fresno Co. & 13 & $8(61)$ & 120 & $10(8)$ & $3(2)$ & $13(11)$ & $1(1)$ & $63(52)$ \\
\hline Kern Co. & 6 & $4(67)$ & 42 & $5(12)$ & $1(2)$ & $6(14)$ & $5(12)$ & $5(12)$ \\
\hline Tulare Co. & 4 & $4(100)$ & 41 & $8(19)$ & 0 & $8(19)$ & 0 & $19(46)$ \\
\hline Total & 41 & $26(63)$ & 401 & $50(12)$ & $9(2)$ & $59(15)$ & $23(6)$ & $103(26)$ \\
\hline \multicolumn{9}{|l|}{ South California } \\
\hline Riverside Co. (desert area) & 17 & $17(100)$ & 156 & $82(52)$ & $2(1)$ & $84(54)$ & 0 & $3(2)$ \\
\hline Total California & 166 & $148(89)$ & 1,735 & $638(38)$ & $180(10)$ & $818(47)$ & $297(17)$ & $115(7)$ \\
\hline
\end{tabular}

a Percentage of the total number of cankers sampled per county.

b Number of vineyards sampled.

c Number of vineyards (and percentage of the total number of vineyards sampled per county) yielding Botryosphaeria spp.

d Total number of cankers collected.

e $\mathrm{A}=$ number of cankers from which a Botryosphaeria sp. only was isolated.

${ }^{\mathrm{f}} \mathrm{B}=$ number of cankers from which a Botryosphaeria sp. was isolated together with other grapevine wood fungal pathogens, such as Eutypa lata, Phomopsis viticola, Phaeoacremonium spp., Phaeomoniella chlamydospora, and other species of ascomycetes.

$\mathrm{g}$ Statistical analyses of the data were conducted with the total number of cankers yielding Botryosphaeria spp. (A + B) from each vineyard sampled in California. 
narda philoprina (Berk. \& Curt.) Van der $\mathrm{Aa}$ and Mycosphaerella pini Rostr. in Monk were used as outgroups for all analyses. In order to determine whether the ITS and $\beta$-tubulin sequence data could be combined, the partition homogeneity test was performed with 1,000 replicates in PAUP. Maximum parsimony for all analyses was performed using the heuristic search option (branch swapping NNI), and 1,000 random addition sequences replicates. Bootstrap values were evaluated using 1,000 replicates to test branch strength. Tree length, consistency index (CI), retention index (RI), rescaled consistency index (RC), and homoplasy index (HI) also were recorded for all analyses. Resulting trees were printed in PAUP version 4.0b10. ITS and $\beta$-tubulin sequences from Botryosphaeria spp. found in California were deposited into GenBank. Botryosphaeria spp. isolated from grapevines in California are maintained in the collection in the Department of Plant Pathology at the University of California, Davis, and representative isolates of each species were placed in the American Type Culture Collection (ATCC).

\section{RESULTS}

Field survey, fungal isolation, and data analyses. Botryosphaeria spp. were found in 148 of 166 vineyards sampled and were isolated from 818 of 1,735 cankers (Table 1). In 638 of 818 cankers yielding Botryosphaeria spp., it was the only fungus isolated from decayed wood tissue; whereas, in 180 cankers, Botryosphaeria spp. were co-isolated with other known grapevine wood fungal pathogens (Table 1). Botryosphaeria was the most abundant fungal genus isolated from grapevine cankers in California, followed by Eutypa lata, Phomopsis viticola, and another currently unidentified Phomopsis spp. Phaeomoniella chlamydospora and various species of Phaeoacremonium occasionally were isolated from cankers as well. Other sporadically isolated fungi from cankers in California were a Clonostachys sp., Truncatella angustata, Alternaria alternata, a Pestalotia sp., and the teleomorphs of Phomopsis spp., Diaporthe helianthi, and D. phaseolorum.

Incidence of Botryosphaeria spp. varied according to grapevine regions. It was significantly greater in south and central

Table 2. Botryosphaeria isolates from Vitis vinifera from California used in this study

\begin{tabular}{|c|c|c|c|c|c|c|}
\hline \multirow[b]{2}{*}{ Species $^{\mathrm{a}}$} & \multirow[b]{2}{*}{ Origin $^{\mathbf{b}}$} & \multirow[b]{2}{*}{ Date collected $^{\mathrm{c}}$} & \multirow[b]{2}{*}{ Isolate } & \multicolumn{2}{|c|}{ GenBank no. } & \multirow[b]{2}{*}{ ATCC no. } \\
\hline & & & & ITS $^{\mathbf{d}}$ & $\beta$-Tubulin & \\
\hline Botryosphaeria australis & Sonoma Co. & 4 September & UCD1314So ${ }^{\mathrm{e}, \mathrm{f}, \mathrm{g}}$ & DQ008323 & DQ008346 & MYA-3698 \\
\hline B. australis & Sonoma Co. & 4 September & UCD1467So ${ }^{e, f, g}$ & DQ233610 & DQ233631 & MYA-3699 \\
\hline B. dothidea & Sonoma Co. & 4 September & $\mathrm{UCD} 1064 \mathrm{So}^{\mathrm{e}, \mathrm{f}}$ & DQ233600 & DQ233621 & \\
\hline B. dothidea & Sonoma Co. & 4 September & UCD1065So ${ }^{e, f, g}$ & DQ233601 & DQ233622 & MYA-3707 \\
\hline B. dothidea & Sonoma Co. & 4 September & $\mathrm{UCD} 1066 \mathrm{So}^{\mathrm{e}, \mathrm{f}, \mathrm{g}}$ & DQ008324 & DQ008347 & MYA-3708 \\
\hline B. dothidea & Mendocino Co. & 4 September & $\mathrm{UCD} 1156 \mathrm{Me}^{\mathrm{e}}$ & DQ233602 & DQ233623 & $\ldots$ \\
\hline B. dothidea & Mendocino Co. & 4 September & UCD1181Me $\mathrm{e}^{\mathrm{e}, \mathrm{g}}$ & DQ008325 & DQ008348 & MYA-3709 \\
\hline B. dothidea & Mendocino Co. & 4 September & $\mathrm{UCD} 1213 \mathrm{Me}^{\mathrm{e}}$ & DQ008326 & DQ008349 & $\ldots$ \\
\hline B. dothidea & Sonoma Co. & 4 September & $\mathrm{UCD} 1333 \mathrm{So}^{\mathrm{e}}$ & DQ008327 & DQ008350 & \\
\hline B. dothidea & Yolo Co. & 5 April & UCD1672Yo $\mathrm{e}^{\mathrm{eff} g}$ & DQ233603 & DQ233624 & MYA-3710 \\
\hline B. lutea & Temecula Valley, Riverside Co. & 5 May & UCD2057Te e,f,g & DQ233604 & DQ233625 & MYA-3700 \\
\hline B. lutea & Temecula Valley, Riverside Co. & 5 May & UCD2089Te $\mathrm{e}^{\mathrm{e}}$ & DQ233605 & DQ233626 & \\
\hline B. lutea & Temecula Valley, Riverside Co. & 5 May & UCD2090Te e,f,g & DQ233606 & DQ233627 & MYA-3701 \\
\hline B. lutea & Temecula Valley, Riverside Co. & 5 May & UCD2098Te $\mathrm{e}^{\mathrm{e}, \mathrm{f}, \mathrm{g}}$ & DQ233607 & DQ233628 & MYA-3702 \\
\hline B. lutea & Temecula Valley, Riverside Co. & 5 May & $\mathrm{UCD} 2103 \mathrm{Te} \mathrm{e}^{\mathrm{e}, \mathrm{f}, \mathrm{g}}$ & DQ233608 & DQ233629 & MYA-3703 \\
\hline B. lutea & Temecula Valley, Riverside Co. & 5 May & UCD2118Te $\mathrm{e}^{\mathrm{e}, \mathrm{f}, \mathrm{g}}$ & DQ233609 & DQ233630 & MYA-3704 \\
\hline B. obtusa & Madera Co. & 3 October & $\mathrm{UCD} 244 \mathrm{Ma}^{\mathrm{e}, \mathrm{f}, \mathrm{g}}$ & DQ008314 & DQ008337 & MYA-3692 \\
\hline B. obtusa & Monterey Co. & 3 November & $\mathrm{UCD} 352 \mathrm{Mo}^{\mathrm{e}, \mathrm{f}, \mathrm{g}}$ & DQ008315 & DQ008338 & MYA-3693 \\
\hline B. obtusa & Fresno Co. & 3 November & $\mathrm{UCD} 465 \mathrm{Fr}^{\mathrm{e}, \mathrm{f}}$ & DQ008316 & DQ008339 & \\
\hline B. obtusa & Kern Co. & 3 December & UCD602Kre,f,g & DQ008317 & DQ008340 & MYA-3694 \\
\hline B. obtusa & Tulare Co. & 3 December & UCD614Tu ${ }^{\mathrm{e}}$ & DQ008318 & DQ008341 & $\ldots$ \\
\hline B. obtusa & Sonoma Co. & 3 December & $\mathrm{UCD} 65 \mathrm{So}^{\mathrm{e}}$ & DQ008319 & DQ008342 & $\ldots$ \\
\hline B. obtusa & Napa Co. & 3 December & $\mathrm{UCD} 666 \mathrm{Na}^{\mathrm{e}}$ & DQ008320 & DQ008343 & $\ldots$ \\
\hline B. obtusa & San Joaquin Co. & 3 December & UCD710SJ $\mathrm{J}^{\mathrm{ef}, \mathrm{g}}$ & DQ008321 & DQ008344 & MYA-3695 \\
\hline B. obtusa & Stanislaus Co & 4 January & $\mathrm{UCD} 770 \mathrm{St}^{\mathrm{e}}$ & DQ008322 & DQ008345 & \\
\hline B. parva & Sonoma Co. & 3 December & $\mathrm{UCD} 642 \mathrm{So}^{\mathrm{e}, \mathrm{f}, \mathrm{g}}$ & DQ008328 & DQ008351 & MYA-3705 \\
\hline B. parva & Sonoma Co. & 3 December & $\mathrm{UCD} 646 \mathrm{So}^{\mathrm{e}, \mathrm{f}, \mathrm{g}}$ & DQ008329 & DQ008352 & MYA-3706 \\
\hline B. parva & Stanislaus Co & 5 January & UCD759St $\mathrm{e}^{\mathrm{e}} \mathrm{f}$ & DQ233611 & DQ233632 & $\ldots$ \\
\hline B. parva & Napa Co. & 3 December & $\mathrm{UCD} 1125 \mathrm{Na}^{\mathrm{e}, \mathrm{f}}$ & DQ233612 & DQ233633 & $\ldots$ \\
\hline B. parva & Sonoma Co. & 3 December & UCD1349So ${ }^{\mathrm{e}, \mathrm{f}}$ & DQ008330 & DQ008353 & $\ldots$ \\
\hline B. rhodina & Coachella Valley, Riverside Co. & 3 November & $\mathrm{UCD} 191 \mathrm{Co}^{\mathrm{e}}$ & DQ008308 & DQ008331 & $\ldots$ \\
\hline B. rhodina & Coachella Valley, Riverside Co. & 3 November & $\mathrm{UCD} 196 \mathrm{Co} \mathrm{e}^{\mathrm{e}, \mathrm{f}, \mathrm{g}}$ & DQ233592 & DQ233613 & MYA-3687 \\
\hline B. rhodina & Coachella Valley, Riverside Co. & 3 November & UCD197Co ${ }^{\mathrm{e}}$ & DQ233593 & DQ233614 & $\ldots$ \\
\hline B. rhodina & Coachella Valley, Riverside Co. & 3 November & $\mathrm{UCD} 202 \mathrm{Co}^{\mathrm{e}, \mathrm{f}, \mathrm{g}}$ & DQ008309 & DQ008332 & MYA-3688 \\
\hline B. rhodina & Coachella Valley, Riverside Co. & 3 November & $\mathrm{UCD} 205 \mathrm{Co}^{\mathrm{e}, \mathrm{f}, \mathrm{g}}$ & DQ008310 & DQ008333 & MYA-3689 \\
\hline B. rhodina & Coachella Valley, Riverside Co. & 3 November & $\mathrm{UCD} 206 \mathrm{Co}^{\mathrm{e}, \mathrm{f}, \mathrm{g}}$ & DQ008311 & DQ008334 & MYA-3690 \\
\hline B. rhodina & Coachella Valley, Riverside Co. & 3 November & $\mathrm{UCD} 220 \mathrm{Co}^{\mathrm{e}, \mathrm{f}, \mathrm{g}}$ & DQ008312 & DQ008335 & MYA-3691 \\
\hline B. rhodina & Madera Co. & 3 October & $\mathrm{UCD} 256 \mathrm{Ma}^{\mathrm{e}}$ & DQ233594 & DQ233615 & $\ldots$ \\
\hline B. rhodina & Kern Co. & 3 December & UCD526Kr ${ }^{\mathrm{e}}$ & DQ233595 & DQ233616 & $\ldots$ \\
\hline B. rhodina & Merced Co. & 5 April & UCD1814Md $\mathrm{Md}^{\mathrm{e}}$ & DQ233596 & DQ233617 & $\ldots$ \\
\hline B. rhodina & Santa Barbara Co. & 5 May & $\mathrm{UCD}_{1962 \mathrm{SB}^{\mathrm{e}}}$ & DQ233597 & DQ233618 & $\ldots$ \\
\hline B. stevensii & Madera Co. & 3 October & $\mathrm{UCD} 288 \mathrm{Ma}^{\mathrm{e}, \mathrm{f}}$ & DQ008313 & DQ008336 & $\cdots$ \\
\hline B. stevensii & Santa Barbara Co. & 5 May & UCD1953SB ${ }^{e, f, g}$ & DQ233598 & DQ233619 & MYA-3696 \\
\hline B. stevensii & Santa Barbara Co. & 5 May & UCD1965SB ${ }^{\mathrm{e}, \mathrm{f}, \mathrm{g}}$ & DQ233599 & DQ233620 & MYA-3697 \\
\hline
\end{tabular}


coast areas than in the other grapevine regions surveyed $(P<0.001)$. High incidence of Botryosphaeria infection from cankers also was found in the north coast, mountain counties, Sacramento Valley, northern San Joaquin Valley, and southern California (desert area). The lowest inci- dence of Botryosphaeria spp. in cankers was found in the southern San Joaquin Valley grapevine region (Table 1).

Regions with a high incidence of Botryosphaeria spp. (north coast, central coast, south coast, mountain counties, and northern San Joaquin Valley) generally had a low rate recovery of $E$. lata from cankers (Table 1). In the other grapevine region with a high incidence of Botryosphaeria infection, Riverside County, E. lata was entirely absent (Table 1). In the southern San Joaquin Valley, the incidences of both Botryosphaeria spp. and E. lata were low

Table 3. Botryosphaeria sequences from GenBank used in the phylogenetic analysis ${ }^{\mathrm{a}}$

\begin{tabular}{|c|c|c|c|c|c|c|}
\hline \multirow[b]{2}{*}{ Isolate $^{\mathrm{b}}$} & \multirow[b]{2}{*}{ Species } & \multirow[b]{2}{*}{ Host } & \multirow[b]{2}{*}{ Collector } & \multirow[b]{2}{*}{ Origin } & \multicolumn{2}{|c|}{ GenBank no. } \\
\hline & & & & & ITS $^{\mathbf{c}}$ & $\beta$-Tubulin \\
\hline CMW $3386^{\mathrm{d}, \mathrm{e}}$ & Botryosphaeria australis & Wollemia nobiles & M. Ivory & Queensland, Australia & AY615165 & AY615149 \\
\hline CMW 6837e & B. australis & Acacia sp. & M.J. Wingfield & Batemans Bay, Australia & AY339262 & AY339254 \\
\hline CMW $6853^{\mathrm{d}, \mathrm{e}}$ & B. australis & Sequiadendron giganteum & M. J. Wingfield & Canberra, Australia & AY339263 & AY339255 \\
\hline CMW $9072^{\mathrm{e}}$ & B. australis & Acacia sp. & J. Roux/D. Guest & Melbourne, Australia & AY339260 & AY339252 \\
\hline CMW 9073 & B. australis & Acacia sp. & J. Roux/D. Guest & Melbourne, Australia & AY339261 & AY339253 \\
\hline CMW $1110^{\mathrm{d}, \mathrm{e}}$ & B. australis & Widdringtonia nodiflora & W. J. Swart & South Africa & AY615166 & AY615150 \\
\hline CMW $1112^{\mathrm{d}, \mathrm{e}}$ & B. australis & W. nodiflora & W. J. Swart & South Africa & AY615167 & AY615151 \\
\hline STE-U 4599d & B. australis & Vitis vinifera & F. Halleen & South Africa & AY343408 & $\mathrm{n} / \mathrm{a}$ \\
\hline WAC $11075^{\mathrm{d}}$ & B. australis & $V$. vinifera & A. Taylor & Western Australia & AY727835 & $\mathrm{n} / \mathrm{a}$ \\
\hline WAC $11337^{\mathrm{d}}$ & B. australis & $V$. vinifera & A. Taylor & Western Australia & AY727836 & $\mathrm{n} / \mathrm{a}$ \\
\hline WAC $11346^{\mathrm{d}}$ & B. australis & $V$. vinifera & A. Taylor & Western Australia & AY727837 & $\mathrm{n} / \mathrm{a}$ \\
\hline CMW $7020^{\mathrm{e}}$ & B. dothidea & Magnifera indica & G. I. Johnson & Australia & AY615191 & AY615178 \\
\hline CMW $7027^{\mathrm{d}, \mathrm{e}}$ & B. dothidea & M. indica & G. I. Johnson & Australia & AY615192 & AY615179 \\
\hline CMW 7780e & B. dothidea & Fraxinus excelsior & B. Slippers & Switzerland & AY236947 & AY236925 \\
\hline CMW 7999d,e & B. dothidea & Ostrya sp. & B. Slippers & Switzerland & AY236948 & AY236926 \\
\hline CMW $8000^{\mathrm{d}, \mathrm{e}}$ & B. dothidea & Prunus sp. & B. Slippers & Switzerland & AY236949 & AY236927 \\
\hline CMW 9075 & B. dothidea & Populus sp. & G. J. Samuels & New Zealand & AY236950 & AY236928 \\
\hline STE-U $5045^{\mathrm{d}}$ & B. dothidea & V. vinifera & G. Marta & Argentina & AY343414 & $\mathrm{n} / \mathrm{a}$ \\
\hline STE-U 5149d & B. dothidea & V. vinifera & A. J. L. Phillips & Portugal & AY343415 & $\mathrm{n} / \mathrm{a}$ \\
\hline CBS $116743^{\mathrm{d}}$ & B. dothidea & Olea europaea & I. Rumbos & Greece & AY786322 & $\mathrm{n} / \mathrm{a}$ \\
\hline CMW $9076^{\mathrm{d}, \mathrm{e}}$ & B. lutea & Malus $\times$ domestica & S. R. Pennycook & New Zealand & AY236946 & AY236922 \\
\hline CMW10309 d,e & B. lutea & $V$. vinifera & A. J. L. Phillips & Portugal & AY339258 & AY339250 \\
\hline CMW10310 d,e & B. lutea & $V$. vinifera & A. J. L. Phillips & Portugal & AY339259 & AY339251 \\
\hline STE-U $3088^{\mathrm{d}}$ & B. lutea & Bukinghamia sp. & P. W. Crous & Australia & AF452555 & $\mathrm{n} / \mathrm{a}$ \\
\hline STE-U 4593 ${ }^{\mathrm{d}}$ & B. lutea & $V$. vinifera & A. J. L. Phillips & Portugal & AY343417 & $\mathrm{n} / \mathrm{a}$ \\
\hline CBS $110299^{d}$ & B. lutea & $V$. vinifera & A. J. L. Phillips & Portugal & AY928043 & $\mathrm{n} / \mathrm{a}$ \\
\hline CMW 7774 d,e & B. obtusa & Ribes sp. & Slippers \& Hudler & New York, United States & AY236954 & AY236932 \\
\hline CMW $7775^{\mathrm{d}, \mathrm{e}}$ & B. obtusa & Ribes sp. & Slippers \& Hudler & New York, United States & AY236953 & AY236902 \\
\hline CMW $8230^{\mathrm{d}, \mathrm{e}}$ & B. obtusa & $\mathrm{n} / \mathrm{a}$ & $\mathrm{n} / \mathrm{a}$ & $\mathrm{n} / \mathrm{a}$ & AY972104 & AY972119 \\
\hline CMW 8232 & B. obtusa & $\mathrm{n} / \mathrm{a}$ & $\mathrm{n} / \mathrm{a}$ & $\mathrm{n} / \mathrm{a}$ & AY972105 & AY972120 \\
\hline STE-U $4581^{\mathrm{d}}$ & B. obtusa & $V$. vinifera & P. Larignon & France & AY343439 & $\mathrm{n} / \mathrm{a}$ \\
\hline STE-U 5037 $\mathrm{d}$ & B. obtusa & $V$. vinifera & A. J.. Phillips & Portugal & AY343446 & $\mathrm{n} / \mathrm{a}$ \\
\hline STE-U $5052^{\mathrm{d}}$ & B. obtusa & $V$. vinifera & J. M. van Niekerk & South Africa & AY343449 & $\mathrm{n} / \mathrm{a}$ \\
\hline WAC $11073^{d}$ & B. obtusa & $V$. vinifera & A. Taylor & Western Australia & AY727842 & $\mathrm{n} / \mathrm{a}$ \\
\hline WAC $11074^{\mathrm{d}}$ & B. obtusa & $V$. vinifera & A. Taylor & Western Australia & AY727843 & $\mathrm{n} / \mathrm{a}$ \\
\hline CBS $112556^{\mathrm{d}}$ & B. obtusa & Pyrus communis & A. J. L. Phillips & Portugal & AY259096 & $\mathrm{n} / \mathrm{a}$ \\
\hline CMW 7798 d,e & B. parva & M. indica & G. I. Johnson & Australia & AY615183 & AY615170 \\
\hline CMW 7799d,e & B. parva & Persea americana & K. G. Pegg & Australia & AY615184 & AY615171 \\
\hline CMW $9077^{\mathrm{d}, \mathrm{e}}$ & B. parva & Actinidia deliciosa & S. R. Pennycook & New Zealand & AY236939 & AY236913 \\
\hline CMW 9079e & B. parva & A. deliciosa & S. R. Pennycook & New Zealand & AY236941 & AY236915 \\
\hline CMW $9080^{\mathrm{d}, \mathrm{e}}$ & B. parva & Populus nigra & S. R. Pennycook & New Zealand & AY236942 & AY236196 \\
\hline CMW 9081 d,e & B. parva & P. nigra & G. J. Samuels & New Zealand & AY236943 & AY236917 \\
\hline STE-U 5253 & B. parva & $V$. vinifera & A. J. L. Phillips & Portugal & AY343477 & $\mathrm{n} / \mathrm{a}$ \\
\hline CBS $110301^{\mathrm{d}}$ & B. parva & $V$. vinifera & A. J. L. Phillips & Portugal & AY259098 & $\mathrm{n} / \mathrm{a}$ \\
\hline CMW 9074, d,e & B. rhodina & Pinus sp. & B. Slippers & Mexico & AY236952 & AY236930 \\
\hline CMW 10130 d,e & B. rhodina & Vitex donniana & J. Roux & Uganda & AY236951 & AY236929 \\
\hline STE-U $4421^{\mathrm{d}}$ & B. rhodina & Vitis vinifera & F. Halleen & South Africa & AY343479 & $\mathrm{n} / \mathrm{a}$ \\
\hline STE-U $5051^{\mathrm{d}}$ & B. rhodina & V. vinifera & M. Gatica & Argentina & AY343483 & $\mathrm{n} / \mathrm{a}$ \\
\hline WAC $7038^{\mathrm{d}}$ & B. rhodina & $V$. vinifera & P. Wood & Western Australia & AY727848 & $\mathrm{n} / \mathrm{a}$ \\
\hline WAC $9853^{\mathrm{d}}$ & B. rhodina & $V$. vinifera & P. Wood & Western Australia & AY727849 & $\mathrm{n} / \mathrm{a}$ \\
\hline WAC $10712^{\mathrm{d}}$ & B. rhodina & $V$. vinifera & P. Wood & Western Australia & AY727850 & $\mathrm{n} / \mathrm{a}$ \\
\hline CMW 7060 d,e & B. stevensii & Fraxinus excelsior & H. A. van der Aa & Netherlands & AY236955 & AY236933 \\
\hline CMW 7776 $6^{\mathrm{d}, \mathrm{e}}$ & B. stevensii & $\mathrm{n} / \mathrm{a}$ & $\mathrm{n} / \mathrm{a}$ & $\mathrm{n} / \mathrm{a}$ & AY972106 & AY972121 \\
\hline CMW $7781^{\mathrm{d}, \mathrm{e}}$ & B. stevensii & $\mathrm{n} / \mathrm{a}$ & $\mathrm{n} / \mathrm{a}$ & $\mathrm{n} / \mathrm{a}$ & AY972107 & AY972122 \\
\hline STE-U 5038 ${ }^{\mathrm{d}}$ & B. stevensii & $V$. vinifera & A. J. L. Phillips & Portugal & AY343484 & $\mathrm{n} / \mathrm{a}$ \\
\hline WAC $11082^{d}$ & B. stevensii & $V$. vinifera & A. Taylor & Western Australia & AY727838 & $\mathrm{n} / \mathrm{a}$ \\
\hline CBS $112553^{d}$ & B. stevensii & $V$. vinifera & A. J. L. Phillips & Portugal & AY259093 & $\mathrm{n} / \mathrm{a}$ \\
\hline
\end{tabular}

${ }^{a} \mathrm{n} / \mathrm{a}=$ Not available at the time of this publication.

b Acronyms of cultures collections: CMW = Culture Collection Forestry and Agricultural Biotechnology Institute, University of Pretoria, South Africa; STE$\mathrm{U}=$ Department of Plant Pathology, University of Stellenbosch, South Africa; WAC = Department of Agriculture Western Australia, Plant Pathogen Collection; and CBS = Centraalbureau Schimmelcultures, Utrecht, Netherlands.

${ }^{c}$ Internal transcribed spacer (ITS).

${ }^{\mathrm{d}}$ Botryosphaeria isolates from GenBank used in the ITS phylogenetic analysis.

${ }^{\mathrm{e}}$ Botryosphaeria isolates from GenBank used in the combined ITS and $\beta$-tubulin phylogenetic analysis. 
but $P$. viticola was isolated at the highest rate of any region in California (Table 1). Only in four counties, Napa, Solano, San Joaquin, and Stanislaus, was the incidence of E. lata significantly higher than Botryosphaeria spp. $(P<0.001$; Table 1$)$.

To test for a relationship between vineyard age and the occurrence of Botryos- phaeria spp., vineyards were divided into three groups: 10 to 20,21 to 30 , and $>30$ years of age. Based on ANOVA, there was no significant relationship between inci-

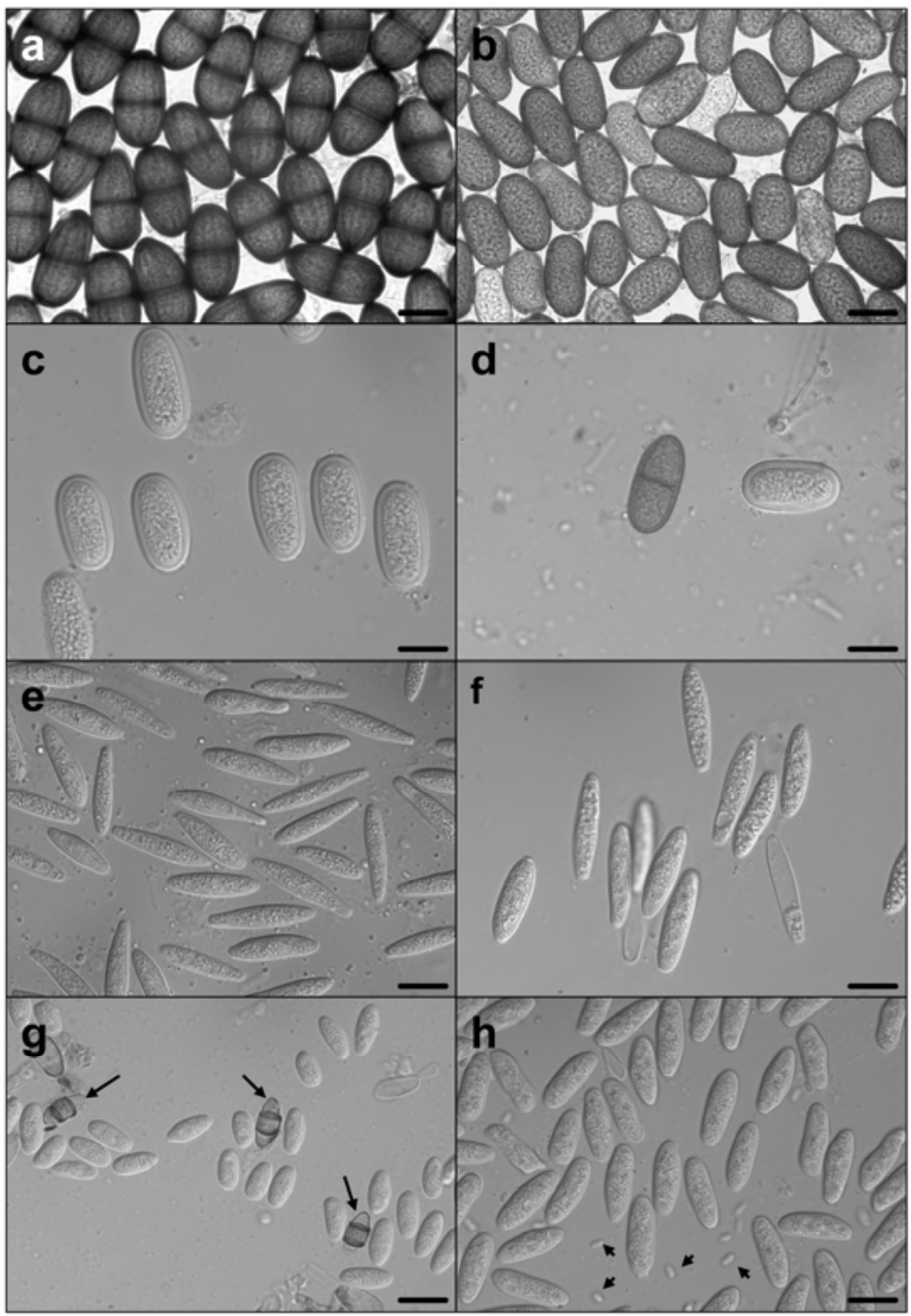

Fig. 2. Conidial morphology of Botryosphaeria spp. Photographs were taken at $\times 100$ (immersion oil) from fruiting bodies (pycnidia) formed in cultures on grapevine wood chips and water agar. a, Mature Conidia of Botryosphaeria rhodina (UCD220Co). B. rhodina shape was oval with rounded and pointed ends. Conidia of $B$. rhodina were hyaline, aseptate, and densely granulate when immature, becoming dark brown, with characteristic longitudinal striations with one central septum when mature. b, Mature B. obtusa conidia (UCD352Mo). Conidia shape of B. obtusa was mostly rounded, but some conidia showed a truncate base. Hyaline when immature, becoming light brown when mature. Aseptate conidia. c, Young, hyaline, thick-walled, one celled, cylindrical with rounded ends $B$. stevensii conidia (UCD1953SB). d, Conidia of B. stevensii sporadically turned brown and developed one septum when mature. e, Hyaline and aseptate conidia of B. dothidea (UCD1065So). B. dothidea conidia were ellipsoidal with rounded and flat ends. f, Fusiform, hyaline, densely granulate, and aseptate $B$. australis conidia (UCD1314So). g, Ellipsoidal with rounded and flat ends conidia of B. parva (UCD642So). Conidia of B. parva were hyaline, densely granulate, and aseptate when immature. Arrows show two septa conidia of $B$. parva, typical characteristic developed of when mature. h, Smooth, fusiform, hyaline, and aseptate conidia of $B$. lutea (UCD2090Te). Arrows show microconidia observed in isolates of $B$. lutea from California. Scale bar $=10 \mu \mathrm{m}$. 
dence of Botryosphaeria spp. and vineyard age $(P=0.807)$. Botryosphaeria spp. were isolated from nearly $45 \%$ of the cankers collected from both the 10- to 20- and 21to 30 -year-old vineyards and from $50 \%$ of cankers in vineyards $>30$ years old. In addition, the effect of wine grape (Cabernet Sauvignon, Cabernet Franc, Chardonnay, Chenin Blanc, French Colombard, Merlot, Shiraz, Sauvignon Blanc, and Zinfandel) and table-raisin grape (Flame, Thompson Seedless, Red Globe, and Perlette) cultivars on the incidence of Botryosphaeria spp. was not significant $(P=$ 0.9883).

Morphological characterization. A total of 44 isolates of Botryosphaeria, representative of the different regions sampled, were divided into groups based on their appearance in culture (Table 2). Eleven isolates were characterized by abundant aerial and fast-growing mycelium that covered an 85-mm-diameter petri plate in $48 \mathrm{~h}$ at room temperature. With age, mycelium became dark green and single or grouped, large (reaching up to 4 to $5 \mathrm{~mm}$ wide), black, globose fruiting bodies (pycnidia), with or without stroma, with central ostioles that were observed after 10 days of incubation at room temperature. All these characteristics were consistent with the description of B. rhodina $(13,37)$. Conidial morphology and DNA analyses also identified these isolates as $B$. rhodina (see below).

A second group of 12 isolates also produced dark-green colonies, but moderate aerial mycelium growth. In 9 of the 12 isolates, single, small, dark-brown and black pycnidia were observed in young cultures ( 7 days old). These isolates also were identified as B. obtusa based on conidial characteristics. The remaining three isolates, which produced no fruiting bod- ies, later were identified as $B$. stevensii based on conidial morphology and sequence data.

A third group of eight isolates initially produced white-cream colonies; however, after 4 days of incubation at room temperature, a characteristic yellow pigment at the center of the colony was observed on the reverse of the culture. This is a typical characteristic of $B$. lutea and $B$. australis according to published descriptions $(20,25,33,37)$. Both species were identified based on conidial morphology and sequence data. Six isolates of $B$. lutea became dark green after 10 days of incubation, whereas two isolates of $B$. australis produced light-gray, pale mycelium, but the reverse of the colony turned dark green. Colony morphology of $B$. australis isolates was well differentiated from $B$. lutea colonies due to the formation of a dense, fluffy, aerial mycelium in the center

Table 4. Conidial dimensions of the Botryosphaeria species from California used in this study and comparison with those reported from preceding studies

\begin{tabular}{|c|c|c|c|c|}
\hline \multirow[b]{3}{*}{ Species, isolate } & \multicolumn{4}{|c|}{ Conidial dimensions of } \\
\hline & \multicolumn{2}{|c|}{ Selected Botryosphaeria spp. in this study } & \multicolumn{2}{|c|}{ Botryosphaeria spp. from previous studies } \\
\hline & Conidial size $(\mu \mathrm{m})^{\mathrm{a}}$ & Mean \pm SD $(\mu \mathrm{m})^{b}$ & Conidial size $(\mu \mathrm{m})$ & Source of data ${ }^{c}$ \\
\hline \multicolumn{5}{|c|}{ Botryosphaeria australis } \\
\hline UCD1314So & $(20-) 25-30 \times(6) 7.3-9.8$ & $25.2 \pm 1.9 \times 7.2 \pm 0.7$ & $(20-) 23-25(-27) \times 4-6^{d}$ & Taylor \\
\hline UCD1467So & $(20.5-) 24.3-31 \times(5.7) 7.3-8.8$ & $24.4 \pm 2.3 \times 7.3 \pm 0.6$ & $(18-) 23-26(-30) \times 5-6(-7.5)$ & Slippers \\
\hline \multicolumn{5}{|l|}{ B. dothidea } \\
\hline UCD1064So & $(21-) 25.5-32 \times(4) 6-8$ & $25.4 \pm 2.4 \times 5.5 \pm 0.8$ & $(18-) 21-28.5(-30) \times(3.5-) 4-4.5(-6)^{\mathrm{d}}$ & Phillips 2002 \\
\hline UCD1065So & $(16-) 23.5-30 \times(5.5) 7-8$ & $23.8 \pm 2.9 \times 6.6 \pm 0.6$ & $17-25 \times 5-7^{\mathrm{d}}$ & Larignon \\
\hline UCD1066So & $(20-) 24-29 \times(5) 7-8$ & $24.1 \pm 2.1 \times 6.6 \pm 0.9$ & $(15-) 20-26(-32) \times(4-) 5-6(-9)$ & Pennycook \\
\hline UCD1181Me & $(19-) 24-29 \times(5) 7.5-9$ & $23.7 \pm 2.3 \times 6.6 \pm 0.7$ & $\ldots$ & $\ldots$ \\
\hline UCD1672Yo & $(19-25.5-30 \times(4) 6-8$ & $25 \pm 3 \times 6.1 \pm 0.9$ & $\ldots$ & $\ldots$ \\
\hline \multicolumn{5}{|l|}{ B. lutea } \\
\hline UCD2057Te & $(14-) 20-25.9 \times(4.6) 6.7-9$ & $20 \pm 2.4 \times 6.6 \pm 0.8$ & $(15-) 18-22.5(-24) \times 4.5-6(-7.5)^{\mathrm{d}}$ & Phillips 2002 \\
\hline UCD2090Te & $(14.3-) 19.3-23.3 \times(5) 6.3-8.8$ & $19.5 \pm 2.1 \times 6.5 \pm 1$ & $(14-) 20-24(-32) \times(5-) 6-7(-9)$ & Pennycook \\
\hline UCD2098Te & $(15-) 20-22.8 \times(5.6) 7.4-9.5$ & $19.7 \pm 1.8 \times 7.4 \pm 0.8$ & $\ldots$ & $\ldots$ \\
\hline UCD2103Te & $(15.3-) 20-27.4 \times(6) 7.6-9.4$ & $20 \pm 2 \times 7.5 \pm 0.8$ & $\ldots$ & $\ldots$ \\
\hline UCD2118Te & $(13.4-) 20.3-23.5 \times(5.6) 7.4-9$ & $20.1 \pm 2.3 \times 7.3 \pm 1$ & $\ldots$ & $\ldots$ \\
\hline \multicolumn{5}{|l|}{ B. obtusa } \\
\hline UCD244Ma & $(17-) 22.5-26 \times(8) 9.5-14$ & $22.3 \pm 2.4 \times 9.4 \pm 1.1$ & $(20-) 21-23(-24) \times 8-10^{\mathrm{d}}$ & Taylor \\
\hline UCD $352 \mathrm{Mo}$ & $(16-) 21-29 \times(9) 11.5-17$ & $20.8 \pm 2.7 \times 11.4 \pm 1.4$ & $(13-) 22-26 \times(9-) 10-13(-15)^{\mathrm{d}}$ & Phillips 2002 \\
\hline UCD465Fr & $(15-) 22-29 \times(7) 9-13$ & $21.5 \pm 3.1 \times 9.1 \pm 1.5$ & $22-26 \times 10-12^{\mathrm{d}}$ & Larignon \\
\hline UCD $602 \mathrm{Kr}$ & $(15-) 23-30 \times(8) 10-14$ & $22.9 \pm 3.2 \times 10 \pm 1.1$ & $20-26 \times 9-12$ & Punithal 1973 \\
\hline UCD710SJ & $(15-) 23-29 \times(7) 10-13$ & $22.3 \pm 3.2 \times 9.9 \pm 1.4$ & $\ldots$ & $\ldots$ \\
\hline \multicolumn{5}{|l|}{ B. parva } \\
\hline UCD642So & $(10-) 14.5-17 \times(5) 7-9$ & $14.3 \pm 1.6 \times 7.1 \pm 1$ & $17-19 \times 5-6$ & Slippers \\
\hline UCD646So & $(11-) 14-17 \times(5) 7.5-9$ & $14 \pm 1.6 \times 6.6 \pm 0.8$ & $(12-) 15-20(-24) \times(4-) 4.5-6(-7.5)^{\mathrm{d}}$ & Phillips 2002 \\
\hline UCD759St & $(11-) 13-19 \times(5) 7-9$ & $13.6 \pm 1.5 \times 7.8 \pm 0.8$ & $(11-14-18(-23) \times 5-7(-10)$ & Pennycook \\
\hline $\mathrm{UCD} 1125 \mathrm{Na}$ & $(11-) 14-23 \times(6) 7-9$ & $14.3 \pm 2 \times 6.8 \pm 0.7$ & $\ldots$ & $\ldots$ \\
\hline UCD1349So & $(11-) 14-18 \times(5.7) 7-9$ & $14.1 \pm 1.7 \times 7 \pm 0.8$ & $\ldots$ & $\ldots$ \\
\hline \multicolumn{5}{|l|}{$B$ rhodina } \\
\hline UCD196Co & $(18-) 20-25 \times(9) 11-14$ & $20.3 \pm 1.5 \times 11.3 \pm 1$ & $(22-) 23-25(-28) \times(15-) 16-18(-20)^{\mathrm{d}}$ & Taylor \\
\hline UCD202Co & $(18-) 22.5-26 \times(10) 12-15$ & $22.2 \pm 1.9 \times 12.2 \pm 1.1$ & $(20-) 24-28(-33) \times(10-) 12-15(-18)$ & Phillips 2004 \\
\hline UCD205Co & $(20-) 25-28 \times(10) 12-16$ & $24.8 \pm 1.8 \times 12.5 \pm 1.4$ & $20-30 \times 10-15$ & Punithal 1980 \\
\hline UCD206Co & $(19-) 23-27 \times(9) 11-14$ & $23 \pm 1.7 \times 11.5 \pm 1.3$ & $\ldots$ & $\ldots$ \\
\hline UCD220Co & $(18-) 23-27 \times(9) 11-14$ & $22.5 \pm 2.5 \times 11.3 \pm 1.3$ & $\ldots$ & $\ldots$ \\
\hline \multicolumn{5}{|l|}{ B. stevensii } \\
\hline UCD288Ma & $(21-) 25-28 \times(10) 13-14.6$ & $24.7 \pm 1.3 \times 12.5 \pm 1$ & $(22-) 24-26(-30) \times 10-14^{\mathrm{d}}$ & Taylor \\
\hline UCD1953SB & $(20-) 24.7-28 \times(10.2) 12.2-14$ & $24.3 \pm 1.7 \times 12.2 \pm 1$ & $(21-) 23-27(-28) \times(10-) 11-12(-13)^{\mathrm{d}}$ & Phillips 2002 \\
\hline UCD1965SB & $(21-) 24.4-28 \times(9.3) 12-14.6$ & $24.3 \pm 1.6 \times 11.9 \pm 1.1$ & $\ldots$ & $\ldots$ \\
\hline
\end{tabular}

a Minimum size, most repetitive value and maximum size for length and width of 60 conidia were recorded from each Botryosphaeria isolate from California selected for conidial dimension.

${ }^{\mathrm{b}} \mathrm{SD}=$ standard deviation.

${ }^{\mathrm{c}}$ Taylor $=$ A. Taylor et al. 2005, Slippers = B. Slippers et al. 2004, Philips $2002=$ A. J. L. Phillips 2002, Larignon = P. Larignon and B. Dubos 2001, Pennycook = Pennycook and Samuels 1985, Punithal 1973 = Punithalingam and Waller 1973, Philips $2004=$ taken from the website of Centro de Recursos Microbiológicos (Faculdade de Ciências e Tecnologia, Universidede Nova de Lisboa, 2829-516 Caparica, Portugal), and Punithal $1980=$ E. Punithalingam 1980 .

d Conidial dimension from Botryosphaeria spp. from Vitis vinifera. 
becoming flat around the edges of the colony. Presence of microconidia in $B$. lutea isolates was another characteristic that differentiated this species from $B$. australis.

The last group, comprising 13 isolates, developed a white aerial mycelium which gradually turned green to dark green after a 4- to 5-day incubation period at room temperature. Of the 12 isolates, 8 formed individual, small, black pycnidia after 3 weeks of incubation at room temperature and were determined to be $B$. dothidea based on conidial morphology from those pycnidia and DNA analysis. For the five remaining isolates, dark, globose pycnidia embedded in stroma tissue were observed after 6 weeks of incubation. Conidial characteristics and DNA sequence data identified isolates in this group as $B$. parva.

All isolates of Botryosphaeria could be separated into two groups based on conidial characteristics. One group was characterized by production of pigmented and thick-walled conidia, and included $B$. rhodina, B. obtusa, and B. stevensii (Fig. $2 \mathrm{a}-\mathrm{d})$. The second group, characterized by the production of hyaline and thin-walled conidia, comprised B. dothidea, B. australis, B. parva, and B. lutea (Fig. 2e-h). Conidial dimensions recorded for Botryosphaeria spp. from California are shown in Table 4, along with previously reported values $(12,20,25-27,34,37)$.

Radial growth values were plotted versus temperature (Fig. 3, only representative isolates shown). All isolates grew over a range of temperatures $\left(10\right.$ to $\left.40^{\circ} \mathrm{C}\right)$, but the optimal temperature varied depending on the species. Botryosphaeria spp. did not grow at $5^{\circ} \mathrm{C}$ and, with the exception of $B$. rhodina, they exhibited only very slight growth at $40^{\circ} \mathrm{C}$ (Fig. 3). The estimated temperatures at which each Botryosphaeria sp. reached the maximum radial growth were $30.8^{\circ} \mathrm{C}$ for both $B$. rhodina and $B$. dothidea, $29.4^{\circ} \mathrm{C}$ for $B$. lutea, $28.2^{\circ} \mathrm{C}$ for $B$. parva, $27.8^{\circ} \mathrm{C}$ for $B$. australis, $26.8^{\circ} \mathrm{C}$ for B. obtusa, and $24.8^{\circ} \mathrm{C}$ for B. stevensii. Growth rates significantly differed between the species of Botryosphaeria at the optimum temperatures $(P<0.001)$ (Fig. 4). Among the Botryosphaeria spp. tested, $B$. rhodina had the highest mycelial growth rate at the optimum temperature after $48 \mathrm{~h}$, whereas $B$. australis exhibited the lowest growth at its optimum temperature (Fig. 4). There was no significant variation between isolates of the same species at each specific temperature. All isolates of $B$. rhodina included in this study started to produce a red pigment (chromogenesis) in the agar or the aerial mycelium after 2 weeks of growth at $35^{\circ} \mathrm{C}$. No chromogenesis was observed in the other Botryosphaeria spp. examined.

Phylogenetic analyses. ITS sequences of Californian Botryosphaeria isolates from grapevine (Table 2) were aligned with GenBank ITS sequences of Botryos- phaeria isolates from grapevine and other hosts (Table 3). Of the 573 nucleotides analyzed, 107 were parsimony informative. Maximum parsimony analyses of the ITS region resulted in one most parsimonious tree (length $=341, \mathrm{CI}=0.839, \mathrm{RI}=0.978$, $\mathrm{RC}=0.820$, and $\mathrm{HI}=0.161$; Fig. 5).

The ITS phylogenetic tree included two well-separated clades. Botryosphaeria spp. having pigmented and thick-walled conidia formed a strongly supported clade, with a bootstrap value of $98 \%$. These species have Diplodia anamorphs and included $B$. rhodina, B. obtusa, and B. stevensii. $B$. rhodina formed a strongly supported clade $(90 \%)$ that was a sister clade to the one containing B. obtusa and B. stevensii (Fig. 5). B. obtusa isolates from California had nearly identical sequences and were grouped together with Australian, French,
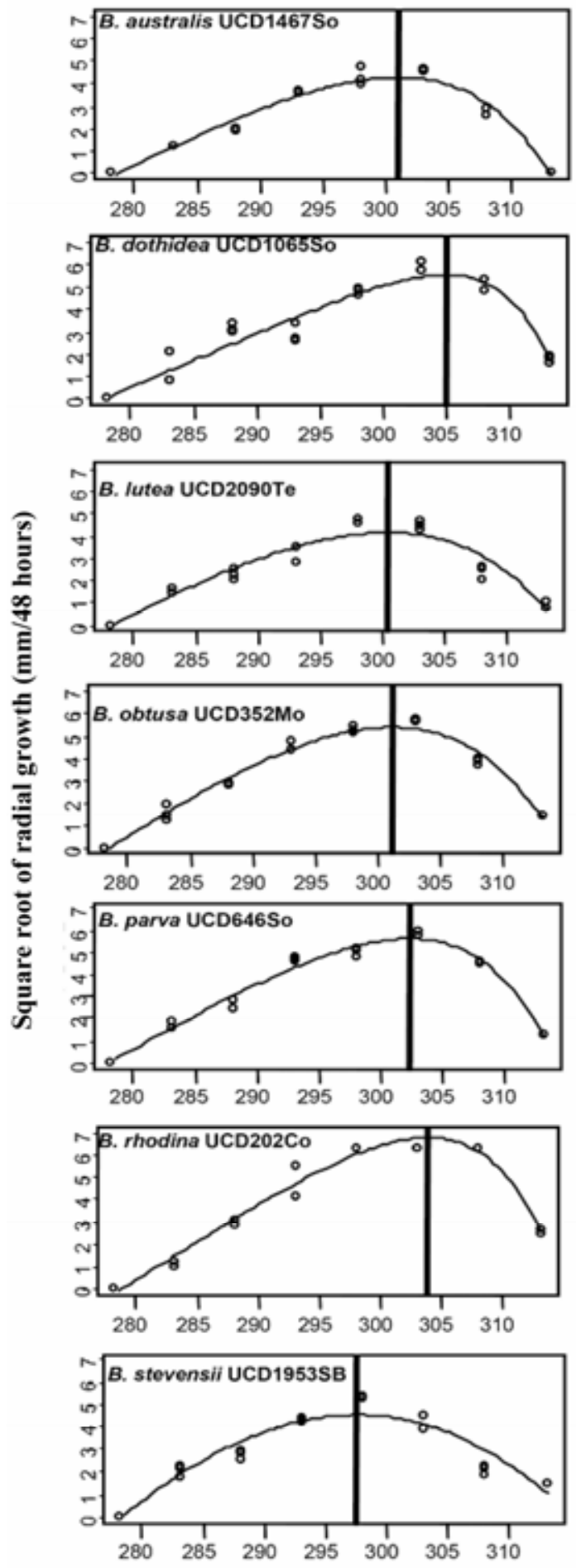

Temperature ( $\left.{ }^{\circ} \mathrm{K}\right)$

Fig. 3. Temperature effect on colony growth of the most representative isolates of Botryosphaeria spp. from California. Black straight lines indicate the estimated optimum growth temperature. 
and Portuguese grapevine isolates (Fig. 5). Botryosphaeria spp. with hyaline and thinwalled conidia resided in a separate clade supported at $60 \%$. These species included B. dothidea, B. lutea, B. australis, and B. parva, which all have Fusicoccum spp. anamorphs (Fig. 5). Within this group, there was no variation in the DNA sequences of $B$. dothidea, which formed a well-supported (100\%) sister group to one comprising B. lutea, B. australis, and $B$. parva (Fig. 5). B. australis showed a close relationship to $B$. lutea. Isolates of $B$. lutea and $B$. australis from California and other areas of the world did not show variation in ITS sequences, except for the B. australis isolate (CMW3386) from Queensland, Australia. Californian isolates of $B$. parva contained no variation in the DNA sequences and were grouped with one isolate from Vitis vinifera from Portugal (Fig. 5).

$\beta$-Tubulin sequences from our grapevine isolates were aligned with 31 GenBank sequences of representative Botryosphaeria spp. (Table 3). Of the 415 nucleotides analyzed, 95 were parsimony informative. The maximum parsimony analyses yielded one most parsimonious tree (length $=320$, $\mathrm{CI}=0.85, \mathrm{RI}=0.965, \mathrm{RC}=0.82$, and $\mathrm{HI}$ $=0.15)$. $\beta$-Tubulin tree topology (Fig. 6) differed from the topology of the ITS tree (Fig. 5) by showing a polytomy at the most basal node of the tree, which separates Botryosphaeria spp. from the outgroup. The $\beta$-tubulin phylogenetic tree included Botryosphaeria spp. with pigmented and thick-walled conidia in a well-separated clade with a bootstrap value of $74 \%$ (Fig. 6). Botryosphaeria spp. with hyaline and thin-walled conidia were separated into two different clades. $B$. dothidea isolates formed a separate clade with bootstrap value of $100 \%$ from all other species with Fusicoccum-type conidia, which resided in a different clade supported at $66 \%$ (Fig. 6). Botryosphaeria isolates with Fusicoccum anamorphs from California had identical $\beta$-tubulin sequences and grouped together

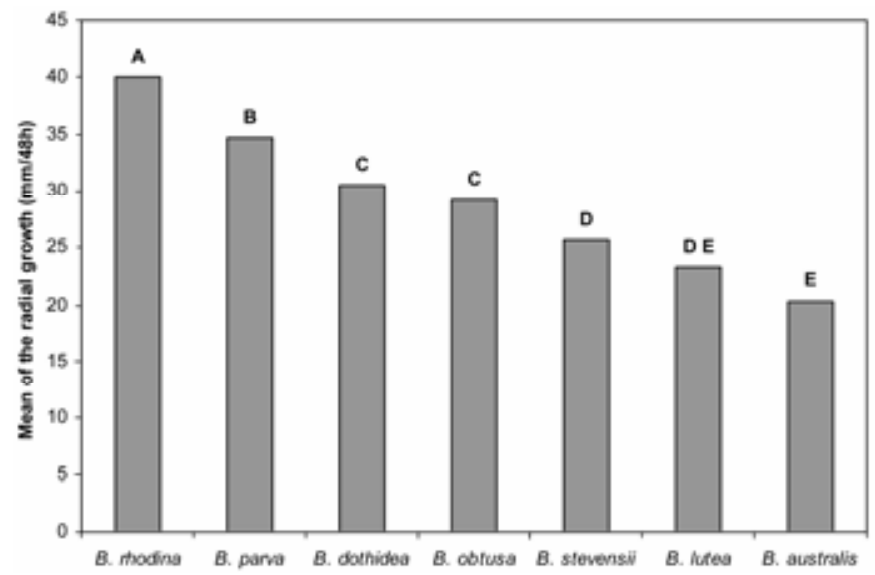

Fig. 4. Mean of the radial growth of the seven Botryosphaeria spp. found in California at the optimum temperature. Mean not represented with the same letter differ significantly according to Tukey's test $(P$ $<0.001)$. with the Culture Collection Forestry and Agricultural Biotechnology Institute, University of Pretoria, South Africa (CMW) collection isolates included in the analysis (Fig. 6).

A partition homogeneity test indicated that the ITS and $\beta$-tubulin datasets could be combined $(P=0.83)$. Of the 987 nucleotides analyzed, 208 were parsimony informative. The maximum parsimony analyses yielded one most parsimonious tree (length $=678, \mathrm{CI}=0.854, \mathrm{RI}=0.971$, $\mathrm{RC}=0.829$, and $\mathrm{HI}=0.146)$. The topology of this tree (Fig. 7) was consistent with the topology of the ITS tree (Fig. 5). Botryosphaeria spp. with Diplodia-type conidia (pigmented and thick walled) formed a highly supported clade $(95 \%)$. Isolates representing individual species within this cluster, B. rhodina, B. obtusa, and $B$. stevensii, were contained in strongly supported individual clades with bootstrap values of 100,99 , and $83 \%$ respectively (Fig. 7). B. obtusa isolates from California grouped together, showing DNA sequence variation from the $B$. obtusa isolates from the CMW collection (Fig. 7). Botryosphaeria spp. with Fusicoccum-type conidia (hyaline and thin walled) composed a separate highly supported clade $(86 \%)$. Within this clade, B. australis, $B$. lutea, B. parva, and $B$. dothidea were all well separated, with bootstrap values of 91, 92, 100, and 100\%, respectively (Fig. 7 ). Based on the combined tree, no variation in the DNA sequences of $B$. dothidea, $B$. lutea, and B. australis isolates was observed, except for both $B$. dothidea (UCD1156Me) and $B$. australis (CMW3386) isolates from California and Australia, respectively (Fig. 7). B. parva isolates from California grouped together, diverging from the $B$. parva isolates from the CMW collection included in the analysis (Fig. 7).

Distribution of Botryosphaeria spp. in California. Seven Botryosphaeria spp. were found in California: B. australis, $B$. dothidea, B. lutea, B. obtusa, B. parva, B. rhodina, and $B$. stevensii. At least one Botryosphaeria sp. was found in all counties sampled in this study (Fig. 8). B. $o b$ tusa was the most frequently isolated species from cankers, followed by $B$. dothidea, $B$. rhodina, $B$. parva, $B$. lutea, $B$. stevensii, and $B$. australis. Differences in geographic distribution of Botryosphaeria spp. throughout California also were apparent. B. dothidea, B. parva, and B. australis primarily were isolated from vineyards in northern California (Fig. 8). B. australis was isolated only from cankers from one vineyard in Sonoma County. None of these species were found throughout the coastal and southern viticulture regions sampled (Fig. 8). B. stevensii was found in only two vineyards located in Madera and Santa Barbara Counties. B. rhodina was found mainly on grapevines from Coachella Valley in Riverside County. However, some isolates of $B$. rhodina also were found in Merced, Madera, Kern, and Santa Barbara Counties (Fig. 8). B. rhodina was not isolated from the northern grapevine areas of California. Isolates of $B$. lutea were only found in five vineyards in Temecula Valley, the other grapevine region sampled within Riverside County.

\section{DISCUSSION}

This study constitutes the first attempt to assess the presence and diversity of Botryosphaeria spp. on grapevine in California. Based on morphological characters and partial sequence analysis of two genes, seven Botryosphaeria spp. were isolated from cankers of grapevines in California from all the major grape-production areas. The associations of $B$. australis, $B$. dothidea, B. lutea, B. obtusa, B. parva, and $B$. stevensii with grapevines in California have not been reported before. Only $B$. rhodina previously was recognized as a pathogen of grapevine in southern California (14).

All the Botryosphaeria spp. found in California have been reported in other grape-growing areas worldwide, and they are associated mainly with a broad range of grapevine symptoms other than cankers. These symptoms include vascular streaking, cane bleaching, graft failure, bud necrosis, limited or no bud burst, and canes with stunted or shortened internodes $(12,25,29,37)$. However, previous studies also have associated Botryosphaeria spp. with cankers. For example, $B$. rhodina and $B$. obtusa frequently have been isolated from cankers in South Africa (41) and Australia (2,37). In Western Australia, $B$. australis and $B$. stevensii also were associated with wedge or half-moon shaped internal lesions of grapevines (37). In Brazil, $B$. dothidea is known to cause "trunk canker" (7) and, in France, B. dothidea, B. obtusa, and B. stevensii also were isolated from sectorial vascular necrosis (12). $B$. obtusa has been reported to cause basal 
canker in cv. Red Globe in Chile (1). The present study also confirms the presence of $B$. parva and $B$. lutea in grapevine cankers.

Vascular cankers and subsequent dieback in California grapevines have been attributed mainly to E. lata and occasionally to Bot canker disease, caused by $B$. rhodina. However, we recovered Botryosphaeria spp. from cankers more often than E. lata, indicating that the former may be a more important cause of grapevine dieback than previously recognized. In part, this may reflect difficulties in distinguishing between vascular cankers caused by Eutypa spp. from those caused by Botryosphaeria spp. $(2,13,38)$. Previous work has indicated that $P$. viticola is not a cause of cankers (18). However, the high incidence of this fungal pathogen isolated from cankers in the southern San Joaquin Valley region underscores its important association with cankers in California.
Whether it is acting as a saprobe or is a weak pathogen on highly susceptible grapevine cultivars, causing the same type of cankers, has not yet been clarified.

Leavitt (13) reported the existence of wedge-shaped cankers caused by $B$. rhodina on 5-year-old vines in the desert area of Coachella Valley in southern California. Four years later, when the vines were 9 years old, dead cordons were observed on most of the vines where cankers

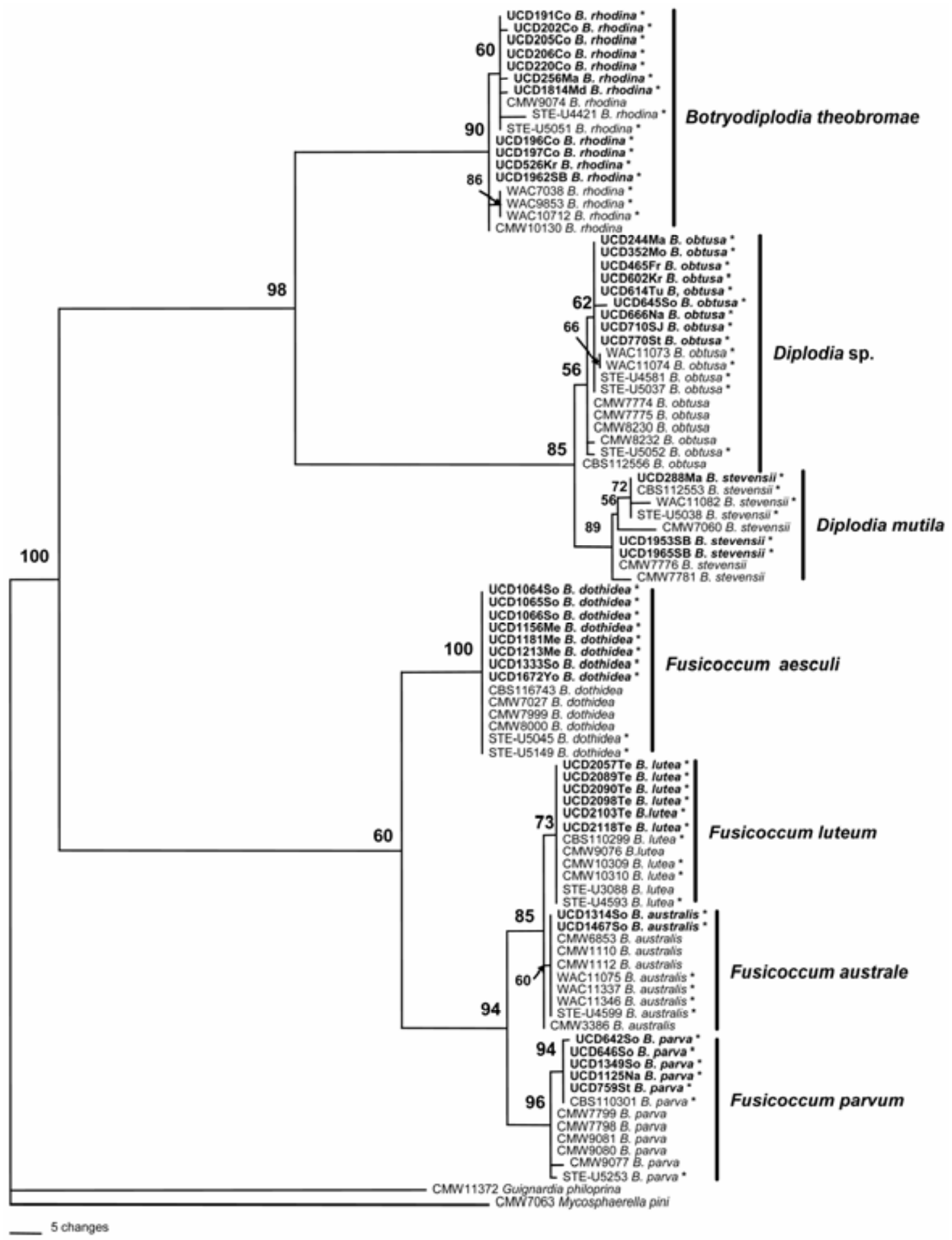

Fig. 5. Most equally parsimonious tree with bootstrap value using 1,000 replicates generated in PAUP 4.0b10 using internal transcribed spacer (ITS)1, 5.8S rDNA region, and ITS2. Asterisks show Botryosphaeria spp. from Vitis vinifera. Anamorph of each Botryosphaeria sp. is shown. 
previously were reported. In the current study, Botryosphaeria spp. all were isolated from cankers with corresponding spur, cordon, or trunk dieback in grapevines 10 or more years old. However, we would expect a survey of vineyards younger than 10 years old also to reveal cankers induced by diverse species of Botryosphaeria.

Susceptibility of grapevine cultivars to some Botryosphaeria spp. has been reported in previous studies. For instance, $B$. dothidea has been shown to be a prevalent and destructive pathogen on muscadine grape ( $V$. rotundifolia) in the southeast of the United States (17). In the Bordeaux region of France, Sauvignon, Cabernet Sauvignon, and Cabernet Franc were the most susceptible cultivars to the disease known as black dead arm, caused by $B$. dothidea, B. obtusa, and B. stevensii, whereas cultivars such as Merlot and Sémillon sustained much lower infection rates (12). In the Australian grapeproduction area of the Hunter Valley, New South Wales, B. obtusa was the fungus most commonly isolated from dieback symptoms in cv. Sémillon. In contrast, results from this study have revealed that all surveyed cultivars were associated with Botryosphaeria spp. Both cultivar groups analyzed in this work, wine grapes and raisin-table grapes, had a similar incidence of infection by Botryosphaeria spp.

Botryosphaeria Ces. \& De Not. constitutes a complex fungal genus with many taxonomic and nomenclatural problems. Because teleomorphs of Botryosphaeria spp. usually are not found in nature and are difficult to obtain in vitro under laboratory conditions, identification of Botryosphaeria spp. in previous studies has been based mainly on morphology of the anamorphs $(4,11,25,37)$. In the present study, none of the teleomorphs of the seven Botryosphaeria spp. were found in any of the samples collected. Anamorph characteristics previously reported $(13,25)$, such as colony and conidial morphology, were useful characters for identification of $B$. rhodina, $B$. obtusa, and B. stevensii isolates from California. However, it remained difficult to separate Botryosphaeria spp. with hyaline conidia, B. australis, B. dothidea, B. lutea, and $B$. parva, based only on conidial morphology.

DNA sequence comparisons allowed us to verify identification of Botryosphaeria spp. from grapevines in California. Results of ITS and combined ITS and $\beta$-tubulin phylogenetic analyses from this study supported previous work that has classified anamorphs of Botryosphaeria in two gen-

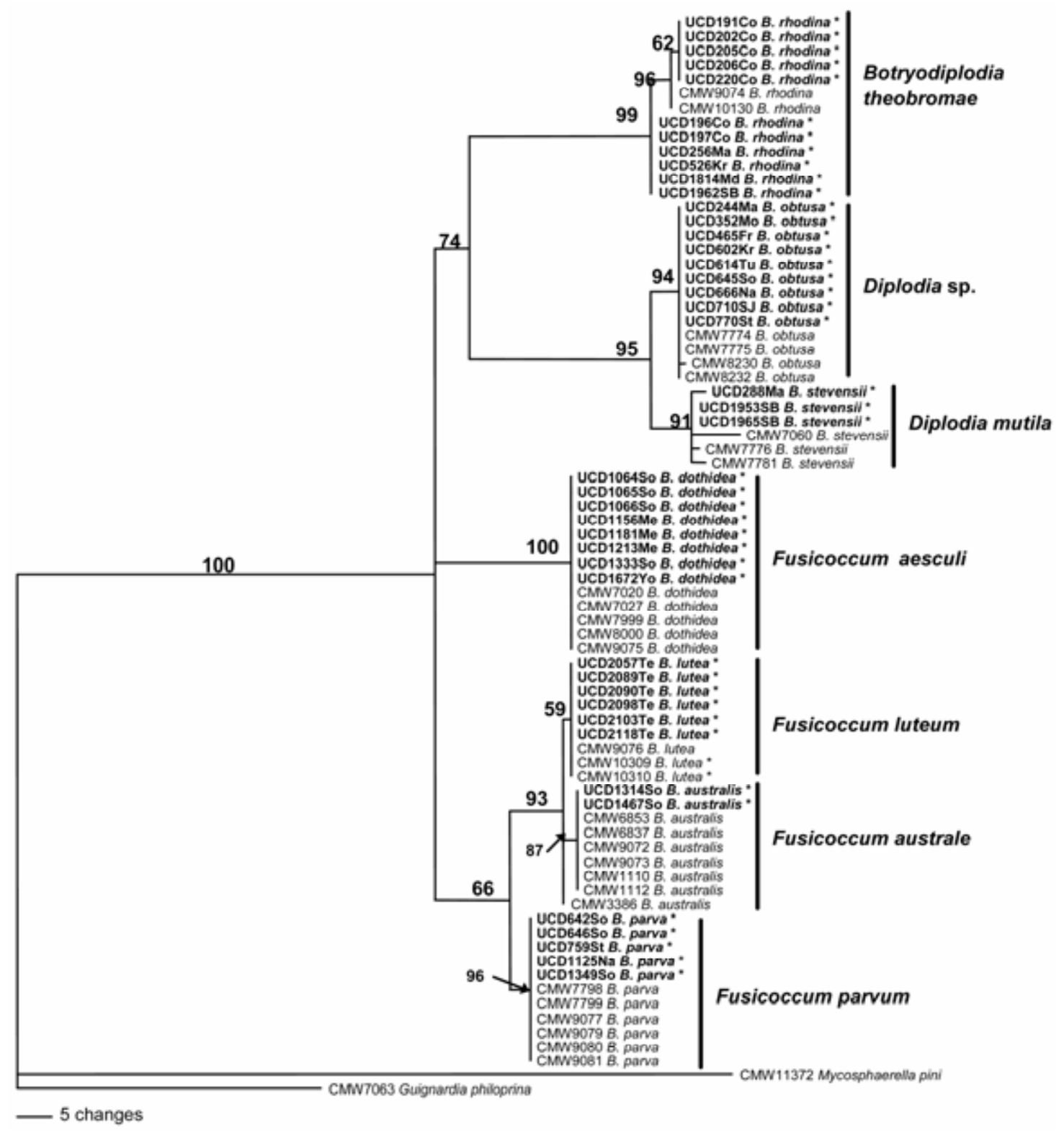

Fig. 6. Most equally parsimonious tree with bootstrap value using 1,000 replicates generated in PAUP $4.0 \mathrm{~b} 10$ from the partial $\beta$-tubulin gene. Asterisks show Botryosphaeria spp. from Vitis vinifera. Anamorph of each Botryosphaeria sp. is shown. 
era on the basis of conidium coloration: Diplodia Fr. and Fusicoccum Corda (4,37,41,45). Although Botryodiplodia theobromae is known as the anamorph of Botryosphaeria rhodina, previous investigations $(4,41,45)$ have suggested that, due to morphological similarities (pigmented, ornamented, and thick-walled conidia), this species should be included under Diplodia Fr. On the other hand, phylogenetic analysis based on $\beta$-tubulin gene se- quences makes the separation of Botryosphaeria spp. between Diplodia and Fusicoccum unclear. The fact that $B$. dothidea formed a separate clade from the other species with Fusicoccum anamorphs indicates uncertainty about phylogenetic relationships within this group.

Our study showed considerable intraspecific variation in both DNA regions examined in both B. rhodina and B. stevensii, and it seems that isolates from California are representative of most of the variants found in a worldwide sampling of these species. The presence of isolates of $B$. rhodina and $B$. stevensii included in this study from different hosts as well as from different geographical locations (Table 3) could explain the variation in the DNA sequences between isolates from California and those from other parts of the world. However, the causes of the intraspecific variation observed between Cali-

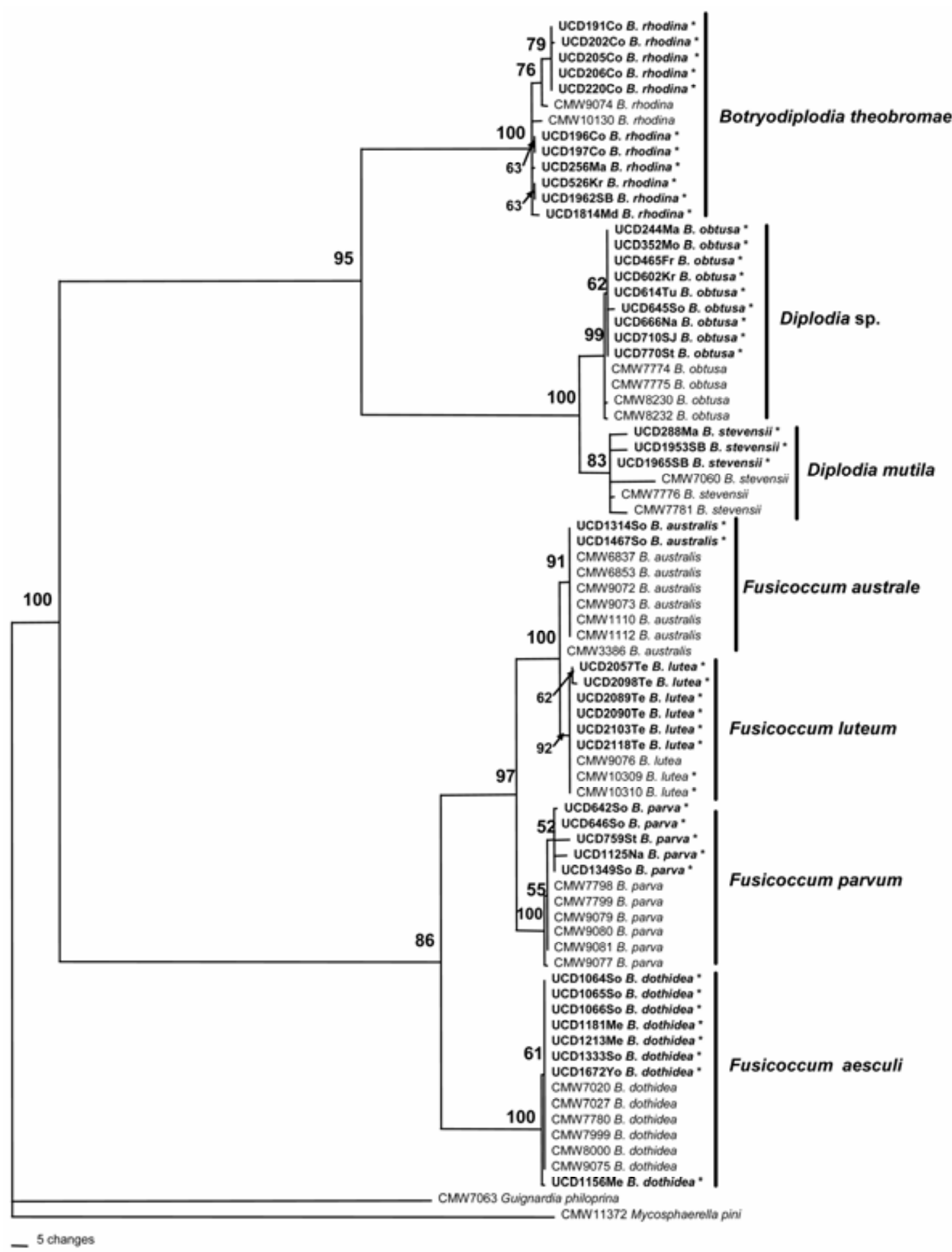

Fig. 7. Most equally parsimonious tree with bootstrap value using 1,000 replicates generated in PAUP 4.0b10 using internal transcribed spacer (ITS)1, 5.8S rDNA region, ITS2, and beta-tubulin partial gene. Asterisks show Botryosphaeria spp. from Vitis vinifera. Anamorph of each Botryosphaeria sp. is shown. 
fornia isolates of these species remain unclear. On the other hand, it is interesting to note that, although isolates of $B$. dothidea, $B$. lutea, and $B$. australis have many different geographic origins and a broad range of hosts, no intraspecific variation was observed. However, much more extensive sampling will be required to determine whether this lack of variation is truly representative of these species worldwide.

Radial growth rates and optimum temperatures obtained in this study for $B$. lutea, B. obtusa, B. parva, B. rhodina, and $B$. stevensii are generally in agreement with data from previous literature (11,13,20,30). However, B. lutea, B. parva, and $B$. stevensii grew at $35^{\circ} \mathrm{C}$ in our temperature study, whereas Pennycook and Samuels (20) reported that B. lutea and $B$. parva failed to grow at this temperature, and Jacobs and Rehner (11) made a similar observation for $B$. stevensii. On the other hand, Sánchez et al. reported slow growth of B. stevensii isolates from oak at $35^{\circ} \mathrm{C}$ (30). The reasons for these discrepancies are unknown, but may simply reflect differences between the isolates studied. The high optimum temperature for $B$. dothidea isolates from California agrees with the

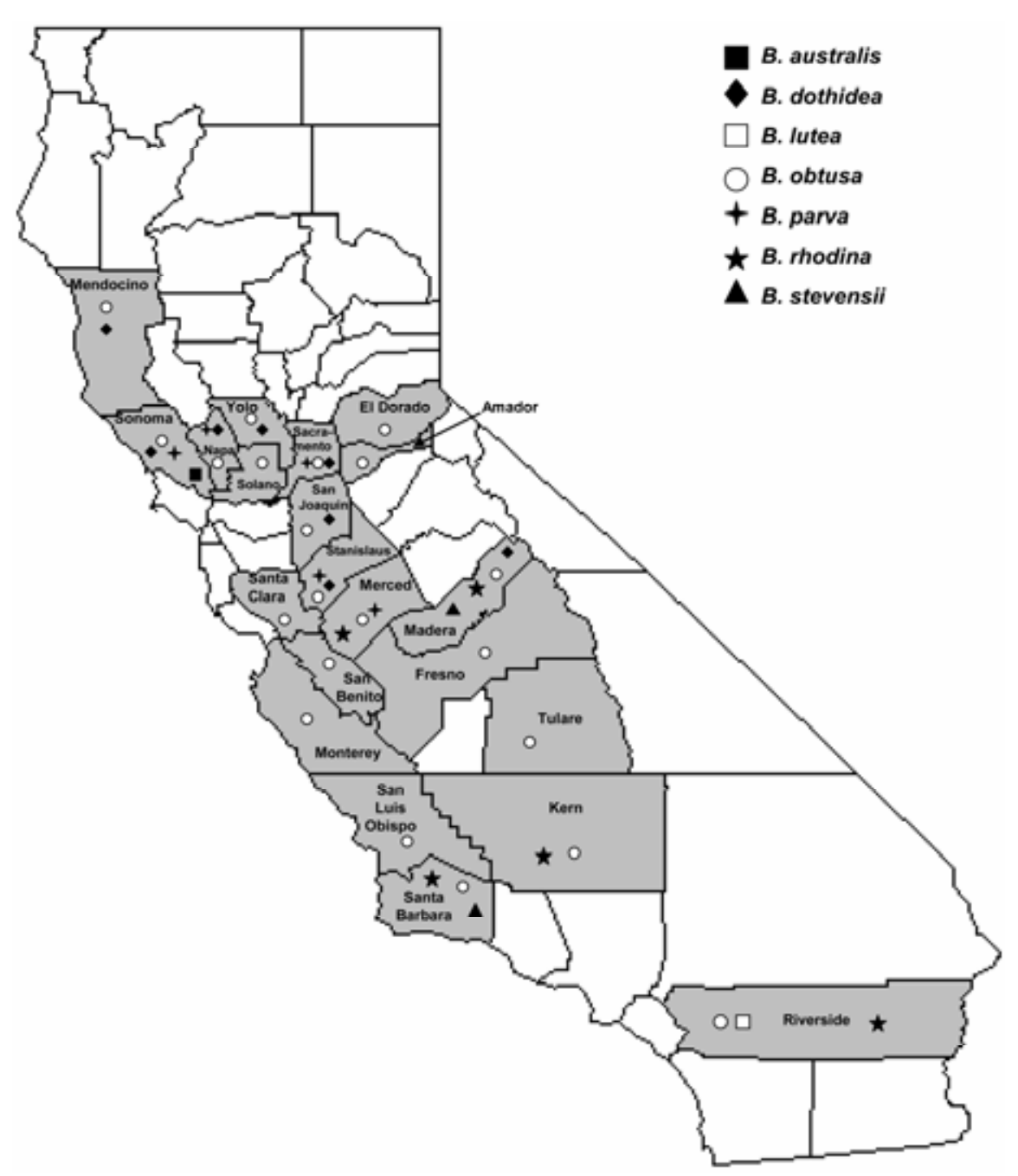

Fig. 8. Geographical distribution of Botryosphaeria spp. on grapevines in California.

data obtained by Jacobs and Rehner (11). However, this result differs with the lower optimum temperatures recorded for $B$. dothidea, $26.1^{\circ} \mathrm{C}$, from the study conducted by Sánchez et al. (30). Chromogenesis, previously reported by other researchers $(13,42)$, was a characteristic observed in the B. rhodina isolates from California when grown at $35^{\circ} \mathrm{C}$ for 2 weeks. The extremely rapid growth rates along with red coloration were useful characters in separating $B$. rhodina from the other species.

The preponderance of $B$. rhodina in the southern part of California, particularly in the desert area of Coachella Valley (Riverside County), and its nonappearance in the northern part of the state could be a consequence of climate differences. Similar results have been reported from a study recently conducted on grapevines in Western Australia, where B. rhodina was isolated only from the warmest area surveyed (37). The geographical distribution of $B$. rhodina observed in this study agreed with the results obtained by Leavitt, in which $B$. rhodina was isolated mainly in the desert area of Coachella Valley and rarely (5\% of the total $B$. rhodina isolates) from the

1502 Plant Disease / Vol. 90 No. 12 northern and southern San Joaquin Valley (13). It is widely known that $B$. rhodina is prevalent in areas with high temperatures, having a broad distribution over tropical and subtropical climates regions (26). The temperatures of the desert production area average 6 to $9^{\circ} \mathrm{C}$ higher than temperatures in the San Joaquin Valley.

A similar outcome was observed with $B$. lutea, which was found only in cankers in Temecula Valley (desert area), but there is insufficient information to explain why $B$. lutea was not isolated in other areas surveyed in California. The assumption that climatic conditions can influence the geographical distribution of Botryosphaeria spp. in California could be reinforced by the fact that $B$. australis, $B$. dothidea, and $B$. parva were found mainly in the northern part of the state. B. australis is a frequent pathogen of native and introduced hosts in the Southern Hemisphere, and may be native to Australia (33). Ours is the first report of $B$. australis on grapevines in the Northern Hemisphere. The frequent recovery of $B$. obtusa and its presence in all grapevine regions in California are consistent with observations in Western Australia, where B. obtusa was the most common and cosmopolitan species isolated from grapevines (37).

Other environmental factors, such as wind, rainfall, and humidity, along with different viticulture practices, may influence the statewide distribution of Botryosphaeria spp. Factors such as rain, wind, source of inocula, or cultivar susceptibility have been shown to be associated with geographical distribution of other grapevine pathogens, such as E. lata and $P$. viticola, in California $(13,18,22)$. However, the effects of these factors on Botryosphaeria spp. on grapevines in California have not yet been studied.

Pathogenicity of the seven Botryosphaeria spp. found in California has been well established on grapevine in other parts of the world, but their relative importance differs by country $(12,37,41)$. Studies designed to characterize the virulence of Botryosphaeria spp. on grapevine in California are now underway.

\section{ACKNOWLEDGMENTS}

We thank E. Petit and F. Trouillas for their support in the phylogenetic analysis; J. Braun, Senior Statistician, Department of Statistics, University of California, Davis, for his support in the data analyses; T. Gordon, R. M. Davis, and J. K. Uyemoto (Department of Plant Pathology, University of California, Davis) for their valuable comments on this manuscript; D. Leonard (Department of Linguistics, University of California, Davis); and UCCE Viticulture Farm Advisors C. Gispert, C. Ingels, D. Hirschfelt, E. Weber, G. McGourty, J. Hashim, L. Bettiga, M. Moratorio, M. Battany, M. Norton, P. Verdegaal, R. Smith, R. Duncan, and S. Vasquez for their valuable assistance and guidance in accomplishing the field collections.

\section{LITERATURE CITED}

1. Auger, J., Esterio, M., Ricke, G., and Pérez, I. 2004. Black dead arm and basal canker of 
Vitis vinifera $\mathrm{cv}$. Red Globe caused by Botryosphaeria obtusa in Chile. Plant Dis. 88: 1286 .

2. Castillo-Pando, M., Somers, A., Green, C. D., Priest, M., and Sriskanthades, M. 2001. Fungi associated with dieback of Semillon grapevines in the Hunter Valley of New South Wales. Aust. Plant Pathol. 30:59-63.

3. Cenis, J. L. 1992. Rapid extraction of fungal DNA for PCR amplification. Nucleic Acids Res. 20:2380.

4. Denman, S., Crous, P. W., Taylor, J. E., Kang, J-C., Pascoe, I., and Wingfield, M.J. 2000. An overview of the taxonomic history of Botryosphaeria, and a re-evaluation of its anamorphs based on morphology and ITS rDNA phylogeny. Stud. Mycol. 45:129-140.

5. El-Goorani, M. A., and El Meleigi, M. A. 1972. Dieback of grapevine by Botryodiplodia theobromae Pat. in Egypt. Phytophatol. Mediterr. 11:210-211.

6. Eskalen, A., Rooney, S. N., and Gubler, W. D. 2002. First report of the pycnidial state of Phaeomoniella chlamydospora, a causal agent of black measles (esca) and Petri disease in California vineyards. (Abstr.) Phytopathology 92:S24.

7. Filho, O. P., Ribeiro, I. J. A., and Kuniyuki, H. 1995. Podridao do tronco da videira (Vitis vinifera) causada por Dothiorella sp., forma anamorfica da Botryosphaeria dothidea. Summa Phytopathol. 21:40-42.

8. Glass Louise, N., and Donaldson, G. C. 1995. Development of primer sets designed for use with the PCR to amplify conserved genes from filamentous Ascomycetes. Appl. Environ. Microb. 61:1323-1330.

9. Gubler, W. D., Rolshausen, P. E., Trouillas, F. P., Úrbez-Torres, J. R., Voegel, T., Leavitt, G. M., and Weber, E. A. 2005. Grapevine trunk diseases in California. Pract. Winery Vineyard (Jan/Feb):6-25.

10. Hewitt, R. W. B. 1994. Diplodia cane dieback and bunch rot. Pages 25-26 in: Compendium of Grape Diseases. American Phytopathological Society Press, St. Paul, MN.

11. Jacobs, K. A., and Rehner, S. A. 1998. Comparison of cultural and morphological characters and ITS sequences in anamorphs of Botryosphaeria and related taxa. Mycologia 90:601-610.

12. Larignon, P., and Dubos, B. 2001. The villainy of black dead arm. Wines Vines 82:86-89.

13. Leavitt, G. M. 1990. The occurrence, distribution, effects and control of Botryodipodia theobromae on Vitis vinifera in California, Arizona and northern Mexico. Ph.D. dissertation, University of California, Riverside.

14. Leavitt, G. M., and Munnecke, D. E. 1987. The occurrence, distribution, and control of Botryodiplodia theobromae on grapes (Vitis vinifera) in California. (Abstr.) Phytopathology 77:1690.

15. Lehoczky, J. 1974. Black dead arm disease of grapevine caused by Botryosphaeria stevensii infection. Acta Phytopathol. Hung. 9:319-327.

16. Luque, J., Martos, S., and Phillips, A. J. L. 2005. Botryosphaeria viticola sp. nov. on grapevines: a new species with a Dothiorella anamorph. Mycologia 97:1111-1121.

17. Milholland, R. D. 1991. Muscadine grapes: some important diseases and their control. Plant Dis. 75:113-117.

18. Moller, W. J., and Kasimatis, A. N. 1981. Further evidence that Eutypa armeniacae, not Phomopsis viticola, incites dead arm symptoms on grape. Plant Dis. 65:429-431.

19. Pascoe, I. 1998. Trunk diseases of grapevines, perspectives from a tour of California. Aust. Graper. Winem. 417:68-71.

20. Pennycook, S. R., and Samuels, G. J. 1985. Botryosphaeria and Fusicoccum species associated with ripe fruit rot of Actinidia deliciosa (Kiwifruit) in New Zealand. Mycotaxon 24:445-458.

21. Petit, E., and Gubler, W. D. 2005. Characterization of Cylindrocarpon species, the cause of black foot disease of grapevines in California. Plant Dis. 89:1051-1059.

22. Petzoldt, C. H., Sall, M. A., and Moller, W. J. 1983. Eutypa dieback of grapevines: ascospore dispersal in California. Am. J. Enol. Vitic. 34:265-270.

23. Phillips, A. J. L. 1998. Botryosphaeria dothidea and other fungi associated with excoriose and dieback of grapevines in Portugal. J. Phytophatol. 146:327-332.

24. Phillips, A. J. L. 2000. Excoriose, cane blight and related diseases of grapevines: a taxonomic review of the pathogens. Phytopathol. Mediterr. 39:341-356.

25. Phillips, A. J. L. 2002. Botryosphaeria species associated with diseases of grapevines in Portugal. Phytopathol. Mediterr. 41:3-18.

26. Punithalingam, E. 1980. Plant diseases attributed to Botryodiplodia theobromae. In: Bibliotheca Mycologica. J. Cramer, Berlin.

27. Punithalingam, E., and Waller, J. M. 1973. Botryosphaeria obtusa. CMI Descriptions of Pathogenic Fungi and Bacteria, No. 394. Commonwealth Agricultural Bureau, Kew, UK.

28. Ratkowsky, D. A., Lowry, R. K., McMeekin, T. A., Stokes, A. N., and Chandler, R. E. 1983. Model for bacterial culture growth rate throughout the entire biokinetic temperature range. J. Bacteriol. 154:1222-1226.

29. Rovesti, L., and Montermini, A. 1987. A grapevine decline caused by Spaheropsis malorum widespread in the province of Reggio-Emilia. Inf. Fitopatol. 37:1-59.

30. Sánchez, M. E., Venegas, J., Romero, M. A., and Phillips, A. J. L. 2003. Botryosphaeria and related taxa causing oak canker in southwestern Spain. Plant Dis. 87:1515-1521.

31. Scheck, H. S., Vasquez, S. J., Gubler, W. D., and Fogle, D. 1998. Young grapevine decline in California. Pract. Winery Vineyard
(May/June):32-38.

32. Siebert, J. B. 2001. Eutypa: The economic toll on vineyards. Wines Vines April:50-56.

33. Slippers, B., Fourie, G, Crous, P. W., Couthino, A. T., Wingfield, B. D., and Wingfield, J. M 2004. Multiple gene sequences delimit Botryosphaeria australis $\mathrm{sp}$. nov. from $B$. lutea. Mycologia 96:1030-1041.

34. Slippers, B., Johnson, G. I., Crous, P. W. Couthino, A. T., Wingfield, B. D., and Wingfield, J. M. 2005. Phylogenetic and morphological re-evaluation of the Botryosphaeria species causing diseases on Mangifera indica. Mycologia 97:99-110.

35. Strobel, G. A., and Hewitt, B. 1964. Time of infection and latency of Diplodia viticola in Vitis vinifera var. Thompson Seedless. Phytopathology 54:636-639.

36. Swofford, D. L. 1999. PAUP*. Phylogenetic Analysis Using Parsimony (*and other methods), version 4.0b4a. Sinauer Associates, Sunderland, MA.

37. Taylor, A., Hardy, G. E. St. J., Wood, P., and Burgess, T. 2005. Identification and pathogenicity of Botryosphaeria species associated with grapevine decline in Western Australia. Aust. Plant Pathol. 34:187-195.

38. Trouillas, F., and Gubler, W. D. 2004. Identification and characterization of Eutypa lepto placa, a new pathogen of grapevine in Northern California. Mycol. Res. 108:1195-1204.

39. Úrbez-Torres, J. R., Peláez, H., Santiago, Y, Martín, C., Moreno, C., and Gubler, W. D 2006. Occurrence of Botryosphaeria obtusa, $B$. dothidea, and $B$. parva associated with grapevine trunk diseases in Castilla y León region, Spain. Plant Dis. 90:835

40. USDA Californian Agricultural Statistics Service 2005. Californian agriculture overview. United States Department of Agriculture.

41. van Niekerk, J. M., Crous, P. W., Groenewald, J. Z., Fourie, P. H., and Halleen, F. 2004. DNA phylogeny, morphology and pathogenicity of Botryosphaeria species on grapevines. Mycologia 96:781-798.

42. Voorhees, R. K. 1942. Live history and taxonomy of the fungus Physalospora rhodina. Fla Agric. Exp. Stn. Bull. 371:91

43. Webster, R. K., Hewitt, W. B., and Polach, F. J. 1969. Studies on Diplodia and Diplodia like fungi. Variation in Diplodia natalensis from grape in California. Hilgardia 39:655-671.

44. White, T. J., Bruns, T., Lee, S., and Taylor, J. 1990. Amplification and direct sequencing of fungal ribosomal RNA genes for phylogenetics. Pages 315-322 in: PCR Protocols, A Guide to Methods and Applications. M. A. Innis, D. H. Gelfand, J. J. Sninsky, and T. J. White, eds. Academic Press, San Diego, CA

45. Zhou, S., and Stanosz, G. R. 2001. Relationships among Botryosphaeria species and associated anamorphic fungi inferred from the analysis of ITS and 5.8S rDNA sequences. Mycologia 93:516-527. 